\title{
Macromolecular crowding links ribosomal protein gene dosage to growth rate in Vibrio cholerae
}

\author{
Alfonso Soler-Bistué ${ }^{1,2}$, Sebastián Aguilar-Pierlé ${ }^{1}$, Marc Garcia-Garceráa ${ }^{3,4,5}$, Marie-Eve Val ${ }^{1}$, Odile Sismeiro ${ }^{6}$, \\ Hugo Varet ${ }^{6}$, Rodrigo Sieira ${ }^{7}$, Evelyne Krin ${ }^{1}$, Ole Skovgaard ${ }^{8}$, Diego J. Comerci ${ }^{2}$, Eduardo P. C. Rocha ${ }^{3,4}$ and \\ Didier Mazel ${ }^{1 *}$
}

\begin{abstract}
Background: In fast-growing bacteria, the genomic location of ribosomal protein (RP) genes is biased towards the replication origin (oriC). This trait allows optimizing their expression during exponential phase since oriC neighboring regions are in higher dose due to multifork replication. Relocation of s10-spc-a locus (S10), which codes for most of the RP, to ectopic genomic positions shows that its relative distance to the oriC correlates to a reduction on its dosage, its expression, and bacterial growth rate. However, a mechanism linking S10 dosage to cell physiology has still not been determined.

Results: We hypothesized that S10 dosage perturbations impact protein synthesis capacity. Strikingly, we observed that in Vibrio cholerae, protein production capacity was independent of S10 position. Deep sequencing revealed that $\mathbf{S 1 0}$ relocation altered chromosomal replication dynamics and genome-wide transcription. Such changes increased as a function of oriC-S10 distance. Since RP constitutes a large proportion of cell mass, lower S10 dosage could lead to changes in macromolecular crowding, impacting cell physiology. Accordingly, cytoplasm fluidity was higher in mutants where S10 is most distant from oriC. In hyperosmotic conditions, when crowding differences are minimized, the growth rate and replication dynamics were highly alleviated in these strains.
\end{abstract}

Conclusions: The genomic location of RP genes ensures its optimal dosage. However, besides of its essential function in translation, their genomic position sustains an optimal macromolecular crowding essential for maximizing growth. Hence, this could be another mechanism coordinating DNA replication to bacterial growth.

Keywords: Ribosomal proteins, Macromolecular crowding, Growth rate, Vibrio cholerae, Bacterial chromosome, Bacterial physiology, Synthetic biology

\section{Background}

Replication, gene expression, and segregation are tightly coordinated with the cell cycle to preserve cellular homeostasis [1, 2]. Genome structure may contribute to integrate these many simultaneous processes occurring

\footnotetext{
* Correspondence: mazel@pasteur.fr

${ }^{1}$ Institut Pasteur, Unité Plasticité du Génome Bactérien, UMR3525, CNRS, Paris, France

Full list of author information is available at the end of the article
}

on the same template. Their relative simplicity and the increasing amount of available data render bacterial genomes ideal models to study this subject [3-6]. Bacterial chromosomes are highly variable in their gene content, but highly conserved in terms of the order of core genes in the chromosomes. Replication begins at a sole replication origin (oriC), proceeding bidirectionally along two equally sized replichores until the terminal region (ter). This organizes the genome along an ori-ter axis that

(c) The Author(s). 2020 Open Access This article is licensed under a Creative Commons Attribution 4.0 International License, which permits use, sharing, adaptation, distribution and reproduction in any medium or format, as long as you give appropriate credit to the original author(s) and the source, provide a link to the Creative Commons licence, and indicate if changes were made. The images or other third party material in this article are included in the article's Creative Commons licence, unless indicated otherwise in a credit line to the material. If material is not included in the article's Creative Commons licence and your intended use is not permitted by statutory regulation or exceeds the permitted use, you will need to obtain permission directly from the copyright holder. To view a copy of this licence, visit http://creativecommons.org/licenses/by/4.0/. The Creative Commons Public Domain Dedication waiver (http://creativecommons.org/publicdomain/zero/1.0/) applies to the data made available in this article, unless otherwise stated in a credit line to the data. 
interplays with cell physiology (Fig. 1a) [4, 5, 7]. For instance, essential genes are overrepresented in the replicative leading strand to avoid head-on collisions between the replication and transcription machineries [8]. Large inversions occur preferentially symmetrically with respect to the ori-ter axis to avoid the emergence of replichore size imbalance $[9,10]$. Recent studies indicate that gene order within the chromosome may play a relevant role in harmonizing the genome structure with cell physiology. Remarkably, key genes coding for nucleoidassociated proteins, RNA polymerase modulators, topoisomerases, and energy production are arranged along the ori-ter axis following the temporal order of their expression during growth phases [11, 12]. In addition, recent studies have showcased an increasing number of traits whose expression is influenced by the genomic position of its encoding genes [13-18]. Notable examples are genes encoding the flux of the genetic information. In fast-growing bacteria, the genes coding for transcription and translation machineries locate near the oriC $[19,20]$. These microorganisms divide faster than the time required for genome duplication. Consequently, chromosomes trigger replication more than once before cytokinesis, overlapping successive DNA duplication rounds, a phenomenon called multifork replication (Fig. 1a). This leads to replication-associated gene dosage gradients along the ori-ter axis during exponential growth (Fig. 1a) [14]. Therefore, it was proposed that the oriC-proximal location of ribosomal and transcription genes allows the recruitment of multifork replication for growth optimization purposes $[5,19,20]$. Thus, the dosage and expression of the aforementioned genes peak during exponential growth phase (Fig. 1a, right) when the transcriptional activity and ribosome numbers increase by 10 - and 15 -fold, respectively [21].

In previous works $[22,23]$, we tackled this issue in Vibrio cholerae, the causative agent of cholera disease. This bacterium harbors a main chromosome (Chr1) of

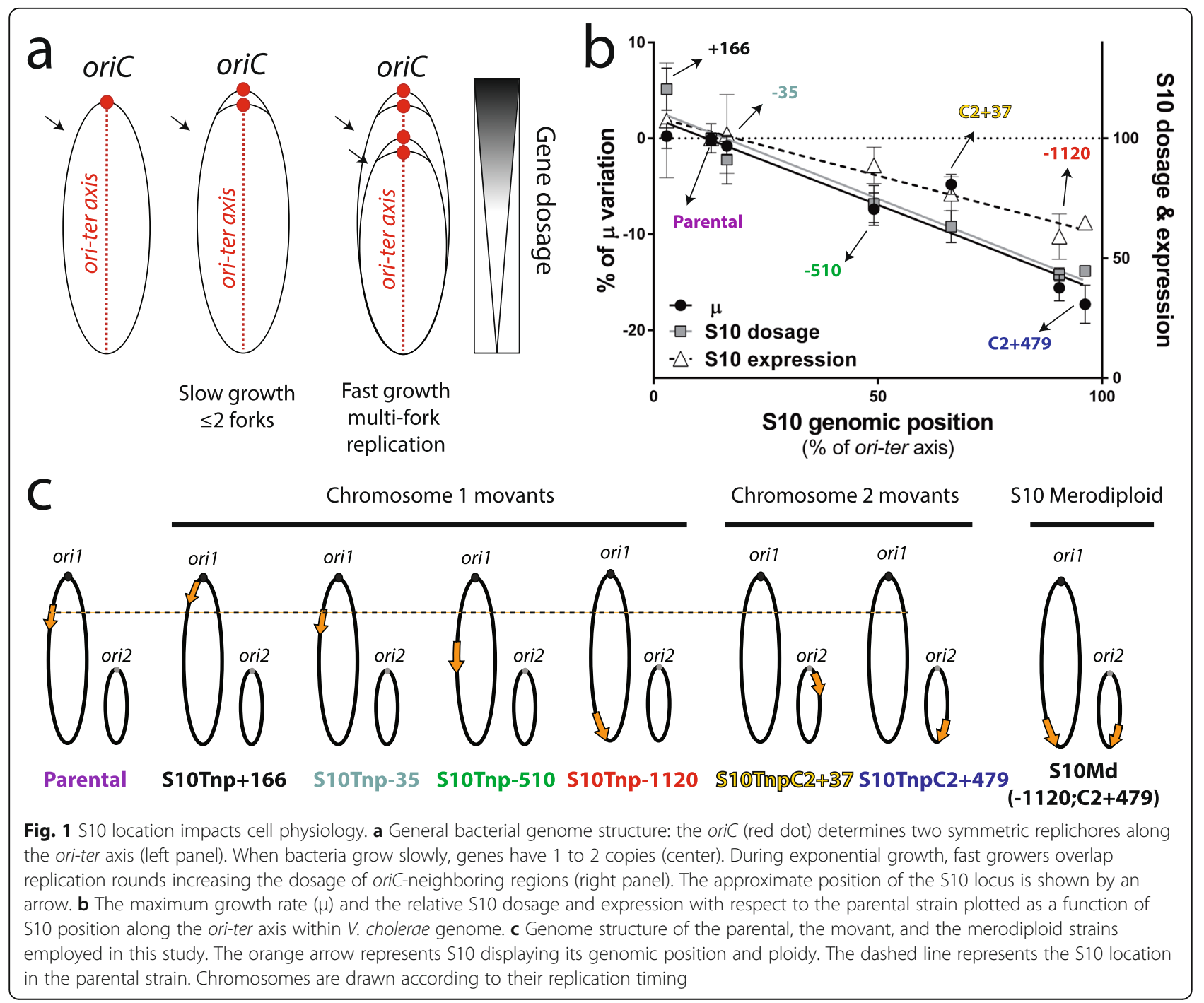


2.96 Mbp and a 1.07-Mbp secondary replicon (Chr2). Their replication is coordinated since the oriC of $\mathrm{Chr} 2$ (ori2) fires after $2 / 3$ of Chr1 duplication has elapsed, finishing the process synchronously $[24,25] . V$. cholerae is among the fastest-growing bacteria displaying particularly high replication-associated gene dosage effects [19]. Its transcription and translation genes map close to the oriC of Chr1 (ori1) [22]. Among them, s10-spc- $\alpha$ (S10) is a 13.4-Kbp locus harboring half of the ribosomal protein genes (RP) located $0.19 \mathrm{Mbp}$ away from ori1 [22]. Using recombineering techniques, we built a set of S10 movants (i.e., isogenic strains where the genomic position of S10 locus is modified) to uncover interplays between the chromosomal position of the locus and cell physiology. We found that its growth rate decreased as a function of the distance between $\mathrm{S} 10$ and ori1 (Fig. 1b, c). Also, S10 genomic location impacted on $V$. cholerae fitness and infectivity [22, 23]. As predicted by bioinformatics $[19,20]$, we showed that oriC proximity of S10 provides optimal dosage to attain the maximal growth capacity [22]. But we also found that S10 position impacts bacterial fitness in the absence of multifork replication suggesting that the RP gene location affects cell physiology even in slow-growing bacteria [23]. In sum, our previous work and the cited examples [14] support the notion that gene order conditions cell physiology, shaping genome structure along the evolution.

Although we proved that the current S10 genomic location maximizes $V$. cholerae fitness [22, 23], we still lack a mechanism explaining this phenomenon. Here, we addressed this issue through the most straightforward hypothesis that is S10 relocation far away from ori1 diminishes ribosome component availability. This, in turn, should reduce ribosomal activity, impacting cell physiology globally through the general impairment of protein synthesis. In this work, we quantified the global protein production in the parental strain and in the most affected derivatives (Fig. 1b, c). Surprisingly, we found no differences in global protein production. RNA and DNA deep sequencing revealed genome-wide alterations in gene transcription and replication dynamics suggesting the existence of global mechanisms linking S10 dosage to cell physiology not linked to protein biosynthesis capacity.

The intracellular milieu has a very high concentration of macromolecules that reaches $400 \mathrm{mg} / \mathrm{mL}$ in Escherichia coli. Consequently, the cytoplasm does not behave as an ideal solution since this large quantity of macromolecules occupies $20-30 \%$ of its volume, which is physically unavailable to other molecules. Such steric exclusion creates considerable energetic consequences, deeply impacting intracellular biochemical reactions. This phenomenon, referred to as macromolecular crowding [26, 27], has received little attention in in vivo systems $[28,29]$. Protein accounts for $\sim 55 \%$ of the bacterial cell mass $[21,26]$, with RP representing one third of them [30]. We hypothesized that S10 expression reduction would lead to lower macromolecular crowding within the bacterial cytoplasm, globally affecting cell physiology [26, 28, 29]. Here, we gathered evidence supporting the idea that S10 relocation mainly impacts cellular physiology of $V$. cholerae by altering cytoplasm homeocrowding (i.e., macromolecular crowding homeostasis) [26].

\section{Results}

\section{S10 relocation does not cause ribosomal activity reduction in normally growing cells}

S10 relocation impacts cell physiology in a dosagedependent manner [22, 23]. However, how S10 dosage reduction affects cell physiology is still unknown. The most straightforward explanation is that a reduction of RP levels upon S10 locus relocation affects ribosome biogenesis leading to a reduction in protein synthesis. To inquire if S10 relocation impairs protein production, we created strains expressing GFP under a strong constitutive promoter into an innocuous intergenic space (Additional File 1: Table S1). The direct quantification of fluorescence allows for estimation of protein production capacity in each strain [31]. First, we followed in time the optical density (OD) and the fluorescence signal of these derivatives. We estimated translation capacity by plotting fluorescence as a function of OD (Fig. 2a). Fluorescence increased exponentially as the OD incremented $\left(R^{2}>0.99\right.$, Additional File 1: Table S2). Although the curves differed slightly between strains, there was no significant correlation between S10 genomic position and GFP production (Pearson's test, $r=0.1, p=0.86$ ). We next subjected cultures of these strains to flow cytometry during early exponential phase, when S10 dosage differences among the movants are maximal. This method allows to simultaneously observe the average GFP production per cell with higher sensitivity and the distribution of fluorescence among the cells in the populations (Fig. 2b). All tested strains showed similar signal levels and the same distribution pattern. In sum, we found no link between GFP production and S10 genomic location.

To confirm that these results were not due to lack of sensitivity, we used the Renilla luciferase (RL) as a reporter of protein synthesis capacity. RL detection shows higher sensitivity than GFP due to lower background, higher signal amplification, and a larger dynamic range, making it suitable to reveal more subtle differences otherwise impossible to differentiate [32]. We built S10 movant strains constitutively expressing $\mathrm{RL}$ at high levels (Additional File 1: Table S1). Again, no differences in the luciferase activity arose between the parental 


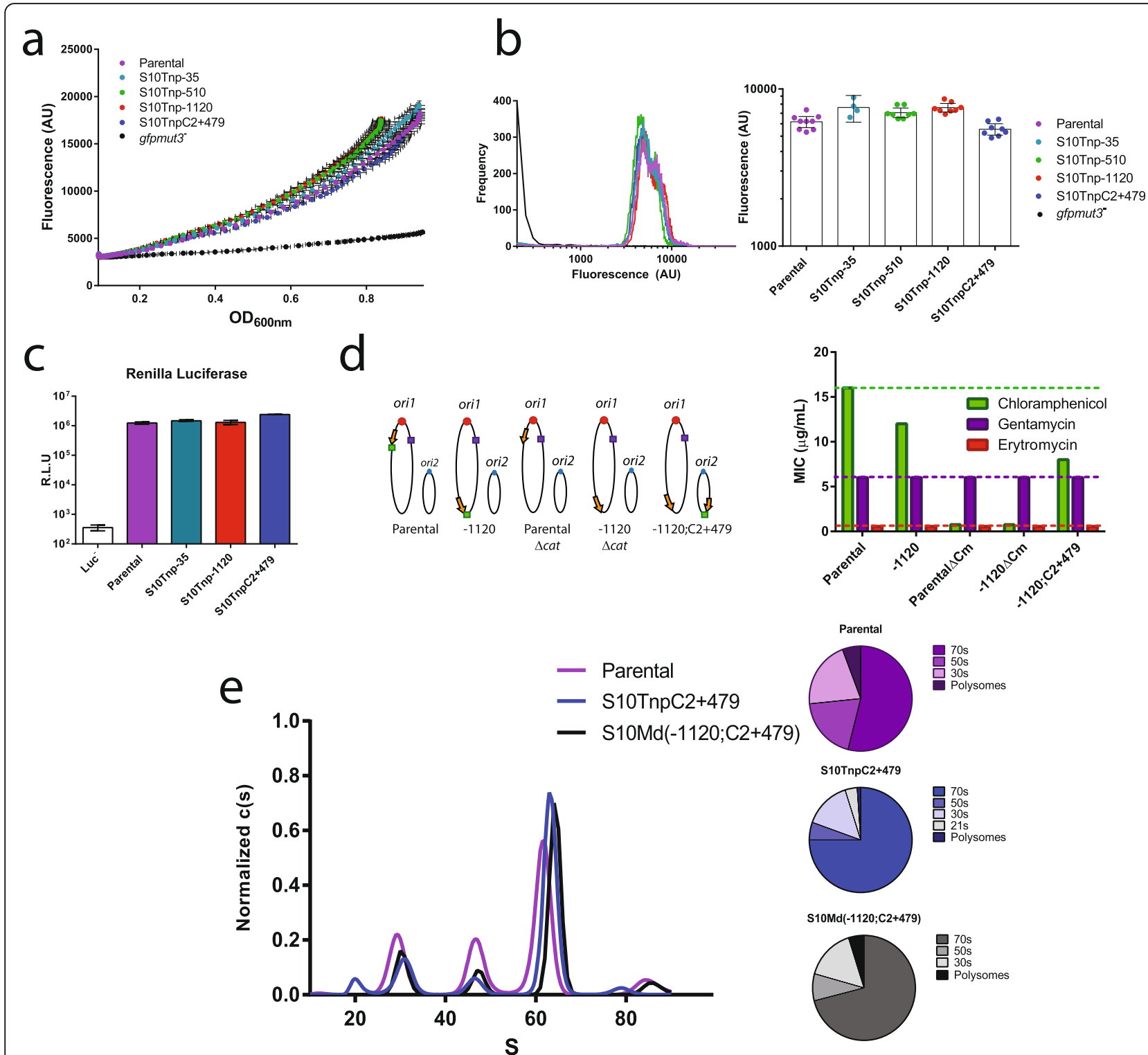

Fig. 2 S10 genomic location does not impact ribosome function in normally growing cells. a The GFP expression and OD $600 \mathrm{~nm}$ of the indicated

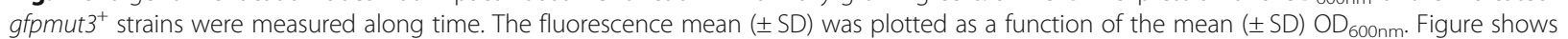
a representative of 3 independent experiments with 4 biological replicates. The parental gfpmut $3^{-}$strain is an autoflourescence/light dispersion control. b The indicated gfpmut ${ }^{+}$strains in early exponential phase were analyzed by FC. Left panel shows the fluorescence signal frequency distribution of the indicated $V$. cholerae strains. A gfpmut $3^{-}$strain was added as negative control. Right panel shows the fluorescence intensity with the 95\% confidence interval (CI). Points represent individual biological replicates obtained along at least 2 independent experiments. c Parental and movant strains bearing RLU in the chromosome (Table S1) were grown until early exponential phase. Then, RL activity, represented as RL units (RLU), was measured in three independent biological replicates for each strain. $\mathbf{d}$ Parental and derivative strains present similar resistance levels to ribosome-targeted antibiotics. On the right panel, chromosomes are represented as in the previous figure. The encoded antibiotic resistance markers are depicted as boxes: $\mathrm{Gm}$ in violet and $\mathrm{Cm}$ in green. Their approximate genomic location is shown in each strain. On the right, the MIC ( $\mu \mathrm{g} / \mathrm{mL})$ for $\mathrm{Cm}, \mathrm{Gm}$, and Er for each depicted strain is shown. e Ribosome profiles for the indicated strains as obtained by AUC. Pie charts quantify polysome, 70s, 50s, and $30 \mathrm{~s}$ fractions for the indicated strains

strain, S10Tnp-35, S10Tnp-1120, and S10TnpC2+479 (Fig. 2c), suggesting similar translation capacity.

It was recently reported that reduction in the number of ribosomes increases the sensitivity to ribosometargeted antibiotics [33]. Hence, we measured the minimum inhibitory concentration (MIC) of chloramphenicol $(\mathrm{Cm})$, gentamicin $(\mathrm{Gm})$, and erythromycin $(\mathrm{Er})$ in the parental and movant strains (Fig. 2d). All the tested mutants derive from a $V$. cholerae isolate sensitive to $\mathrm{Er}$ and harboring $\mathrm{Gm}$ resistance gene 
(Additional File 1: Table S1). Strains that only differed in the genomic location of S10 had their growth inhibited at the same $\mathrm{Er}$ and Gm concentrations (Fig. 2d) suggesting no differences in ribosomal numbers. In parallel, the parental, S10Tnp-1120, and S10Md(-1120;C2+479) strains harbor the $\mathrm{Cm}$ resistance gene (cat) linked to the S10 locus; therefore, the location of the resistance gene differed among them (Fig. 2d). Cm resistance was higher in the parental strain when cat is closer to the ori1 and lower in S10Tnp-1120 and S10Md(-1120;C2+479) when the resistance marker is nearby the ter 1 region. Hence, as in other genetic systems [34], Cm sensitivity varied according to cat genomic location independently of S10 copy number (compare S10Tnp-1120 to S10Md(-1120; $\mathrm{C} 2+479)$ ). Therefore, even though this assay is sensitive enough to capture the effects caused by differences in cat genomic location, it showed no antibiotic susceptibility differences related to S10 genomic location. The lack of effects of S10 relocation on MIC when using any of the three different ribosome-targeting antibiotics, possessing different tolerance levels, suggests that the number of ribosomes is not affected by the genomic location of S10.

\section{S10 genomic location causes changes in maximum GFP synthesis capacity}

Since we did not detect differences in the ribosomal activity with previous approaches, we measured GFP production at the single cell level using fluorescence recovery after photobleaching (FRAP). In this assay, the full area of individual cells expressing GFP was photobleached and followed over time for at least $5 \mathrm{~min}$. Then, we quantified the percentage of fluorescence recovery. This allows comparing the maximum capacity of protein synthesis between strains. In the parental strain, 95\% of the cells displayed a recovery of at least $20 \%$ (mean $=$ $53.8 \%, n=108$ ) of the initial signal after $3 \mathrm{~min}$, to reach a plateau until the end of the observation (Additional File 1: Fig. S1a). The addition of $\mathrm{Cm}$ up to the MIC significantly inhibited the fluorescence increase (mean $=15.8 \%, n=21, p<0.0001$ ), suggesting that signal recovery corresponds to GFP re-synthesis. Meanwhile, we observed a significantly lower average recovery in the most physiologically affected movants S10Tnp-1120 (20.1\%, $n=42, p<0.0001)$ and S10TnpC2+479 (25.8\%, $n=82, p<0.0001$ ) (Additional File 1: Fig. S1b), suggesting that they produced less GFP. Therefore, the parental strain displayed a higher maximum protein synthesis capacity than the most affected S10 movants.

S10 relocation alters the ribosomal sedimentation profile Reduction in RP expression can lead to problems in ribosome assembly due to modifications in the stoichiometry of its components. To detect alterations in ribosome assembly, reflected in changes in ribosomal subunits composition, we performed ribosome preparations followed by analytical ultracentrifugation (AUC) in the parental and the physiologically impaired S10TnpC2+479 strain. We also analyzed a merodiploid strain where most of the growth deficiency is rescued but still displays a reduced growth $(\mathrm{S} 10 \mathrm{Md}(-1120$; $\mathrm{C} 2+479)$ ) [22]. We expected that growth impairment would correlate with a reduction in the proportion of assembled ribosomes (i.e., the 70s peak), when compared to free ribosomal subunits ( $30 \mathrm{~s}$ and $50 \mathrm{~s}$ peaks). Figure $2 \mathrm{e}$ shows that parental strain displayed a $53.97 \%$ of the signal in the peak corresponding to the 70 s while 50 s and 30 s peaks represented 19.4 and $20.8 \%$, respectively. In the S10TnpC2+479 movant, we observed an increase in the 70 s proportion to the $75.85 \%$ of the signal while the free ribosomal subunits lowered to $5.5 \%$ and $14.8 \%$ of the signal for 50 and 30s subunits, respectively. In the S10Md(-1120;C2+479) strain, showing an intermediate growth phenotype, 70s, 50s, and 30s represented 71\%, $8.3 \%$, and $15.8 \%$ of the signal, respectively. Our data shows that a reduction in S10 expression led to an increase of the proportion of assembled ribosomes and a reduction of free ribosomal subunits. Therefore, movant strains might compensate lower S10 expression engaging more free ribosomal subunits into translation. This could explain the relatively low impact of S10 relocation on translation capacity.

\section{Dosage reduction of S10 non-ribosomal genes does not impact cell physiology}

Since reduction of protein biosynthesis upon S10 relocation was mild, we reasoned that it cannot explain the drastic changes observed in fitness and growth rate $(\mu)$. Meanwhile, S10 harbors genes not related to ribosome biogenesis: rpoA, encoding for the $\alpha$-subunit of RNA polymerase, and $\sec Y$, which codes part of the Sec translocon [35], essential for protein export. We wondered whether dosage reduction of rpoA and/or sec $Y$ could contribute to the phenotype caused by $\mathrm{S} 10$ relocation by provoking a reduction of the transcription rate and/or by hampering the normal protein export process. To test this, we cloned rpoA and $\sec Y$ on a low copy number plasmid with inducible expression. The parental strain (Additional File 1: Table S1, Parental) and the two most affected movants, S10Tnp-1120 and S10TnpC2+479, were transformed with either of these plasmids or the empty vector. Next, the $\mu$ of the transformed strains was determined through automated growth curves. If lower RNAP and/or translocon activity was involved in the observed phenotypes, growth rate differences between the parental and movant strains should lessen or disappear upon rpoA and $\sec Y$ overexpression. Results on Additional File 1: Fig. S2 show that the growth rate was 
significantly lower in the movants compared to the parental strain independently of the genes expressed on the plasmid carried. Since the plasmids expressing rpoA or $\sec Y$ did not rescue the growth defect, the impact of S10 relocation on cell physiology results from dosage reduction of RP genes within the locus.

\section{Transcriptome analysis of the movant strain set}

The physiological effects of S10 relocation are due to dosage reduction of RP genes. Changes in translation were not enough to explain the observed physiological effects. Hence, we reasoned that alternative mechanisms must be involved. To detect genes whose transcription was affected by S10 relocation and search for metabolic pathways responding to RP dosage alterations, we characterized the full transcriptome of the following: S10Tnp-35, the movant in which S10 was slightly moved presenting no phenotype, and the physiologically impaired strains S10Tnp-510, S10Tnp-1120, and
S10TnpC2+479 (Fig. 1b). We collected the samples in fast-growing conditions during exponential phase ensuring maximal S10 dosage differences, and then, we compared each movant's transcriptome to the one of the parental strain.

We first looked at the read coverage along the chromosomes, a parameter accounting for the genome-wide transcriptional activity. Surprisingly, we observed that the transcription of the ori1 region slightly decreased as a function of the distance between S10 and ori1 (Fig. 3a and Additional file 1 Fig. S3 and Table S3).

\section{Replication dynamics are altered in the most affected movants}

Given that a specific mechanism regulating the expression of such a wide genomic region seems unlikely, we wondered if the change in the expression of ori1 region was linked to changes in global replication pattern. To assess this, we studied the replication dynamics of the

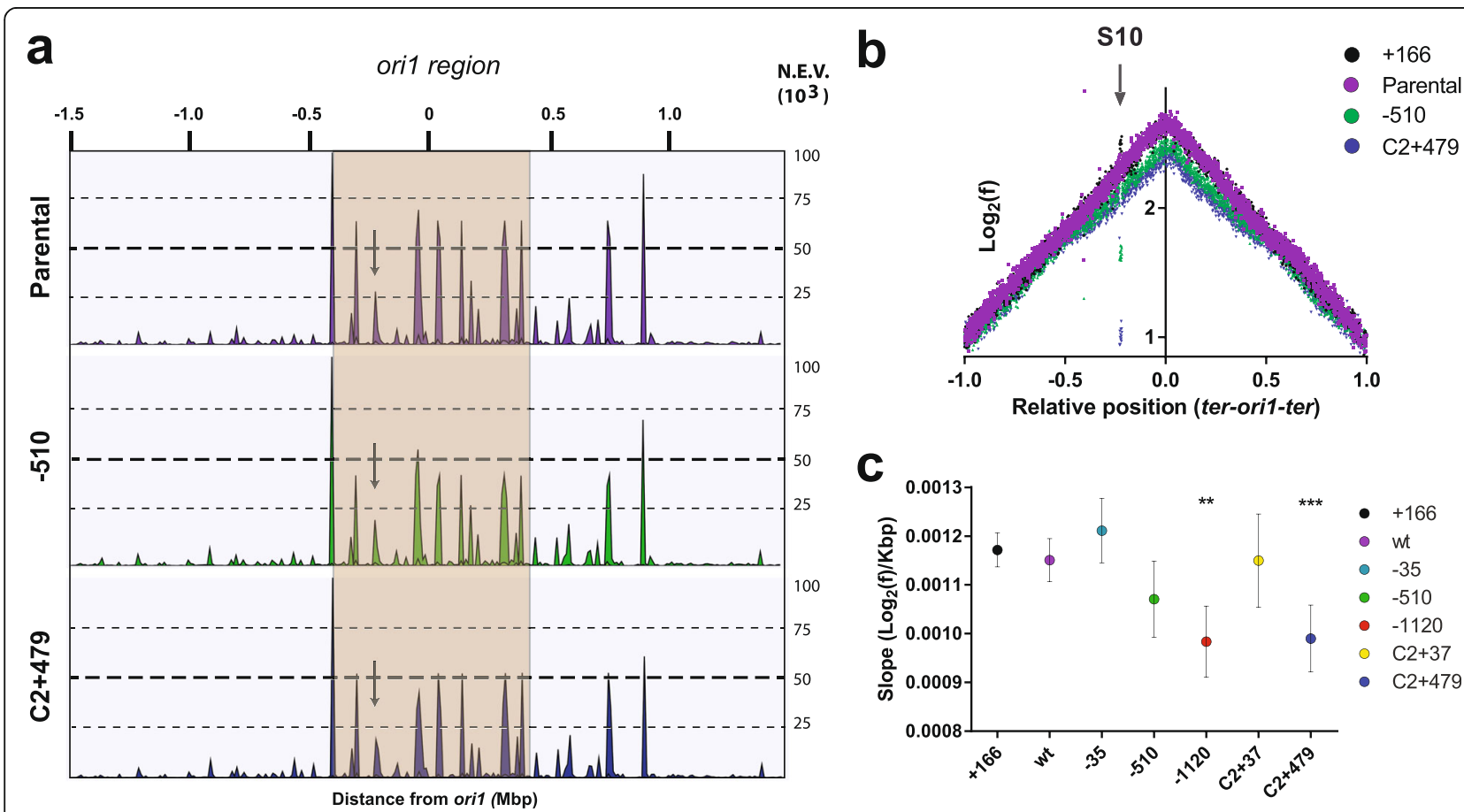

Fig. 3 Genome-wide transcription and replication activity along the genome. a Transcriptional activity across Chr1. RNA-seq reads were mapped along the Chr1 of $V$. cholerae. The histograms represent mapped read normalized to the genome-wide total volume along both replichores in ter1-ori1-ter1 order. Normalized expression values (NEV) are shown along the distance from ori1 in megabase pair which is shown on top. Each graph represents one strain: parental (purple), S10Tnp-510 (green), and S10TnpC2+479 (blue). The plots of the whole strain set are in Fig. S4. The 400-Kbp flanking ori1 are highlighted in orange. The arrow indicates the peak corresponding to the S10 locus. $\mathbf{b}$ MFA profiles are obtained by plotting the $\log _{2}$ frequency of reads (normalized against reads from a stationary phase of a parental strain control) at each position in the genome as a function of the relative position on the $V$. cholerae main chromosome with respect to ori (to reflect the bidirectional DNA replication) using 1000-bp windows. Results for the parental (purple), the S10Tnp+166 (black), the S10Tnp-510 (green), and the S10TnpC2+479 (blue) movants show their differences in read coverage. The arrow highlights the S10 position in the abscissa, reflecting dosage alterations. c S10 relocation effect on replication dynamics was quantified by averaging the obtained slope for each replichore for at least 4 independent MFA experiments in fast-growing conditions. Results are expressed as the mean slope with $95 \% \mathrm{Cl}$. Statistical significance was analyzed by one-way ANOVA two-tailed test. Then, Tukey's test was done to compare the mean values obtained for each strain. Statistically different slopes are indicated as follows: ${ }^{* *} p<0.01$ and ${ }^{* * *} p<0.001$ 
genome of the whole strain set using marker frequency analysis (MFA) in fast-growing conditions. For this, we aligned genomic DNA reads from exponentially growing cells of each strain to the $V$. cholerae genome. For each replicon, there is a linear relationship between the $\log _{2}$ number of reads covering the locus and its genomic position between the oriC and the ter [36] (Fig. 3b). This allows for robust quantification of replication dynamics across the bacterial genome with unprecedented resolution of replication fork speed and the ori and ter region locations [25, 36-38]. To better quantify these differences, we calculated the average slope $\left(\log _{2}\right.$ (frequency)/Kbp) along both replichores, which estimates the replication speed for each strain (Fig. 3c). MFA analysis revealed significant differences in replication dynamics across the strain set. The parental strain, the S10Tnp+166, and the S10Tnp-35 displayed a similar slope (Additional file 1: Table S4). Conversely, the most affected movants, S10Tnp-1120 and S10TnpC2+479, where S10 was relocated at the termini of Chr1 and Chr2, showed a significantly lower slope $(p<0.01$, Fig. 3b, $c$ and Table S4). S10Tnp-510 and S10TnpC2+37 displayed an intermediate value not significantly different from either group. Coincidentally, the calculated slope closely correlated to the S10 locus genomic position $(r=$ $-0.78, p<0.05)$, its dosage $(r=0.8, p<0.05)$, the ori1/ ter1 ratio $(r=0.91, p<0.005)$, and $\mu(r=0.9, p<0.01)$ (Additional file 1: Fig. S5). This suggests that the genomic location of S10 impacts DNA replication activity, slowing down replication when S10 is far from ori1. These data (Fig. 3b, c and Additional file 1: Table S4) indicate that DNA coverage decreases at the ori1 region with increasing ori1-S10 distance matching the changes in transcriptional coverage observed in RNA-seq data.

\section{Differentially expressed genes upon S10 relocation}

We next analyzed the transcriptomic data to find which genes and pathways differentially transcribed with respect to the parental strain in S10Tnp-35 and in the affected movants S10Tnp-510, S10Tnp-1120, and S10TnpC2+479 (Fig. 1b, c).

Using volcano plots, we analyzed the statistical significance of the changes in transcription of each gene ($\log _{10}(p$ value $\left.)\right)$ as a function of its transcriptional $\log _{2}$ of fold change $\left(\log _{2}(\mathrm{FC})\right)$ compared to the parental strain. We observed more transcriptionally altered genes with higher distances between the S10 locus and ori1 (Fig. 4a). S10Tnp-35, a strain presenting no phenotype used as a control of the neutrality of the relocation process, displayed only 8 genes with significant $(p<0.05)$ transcriptional change (Table 1, Additional file 2: Dataset S1). S10Tnp-510, displaying a $\sim 7 \%$ growth rate reduction
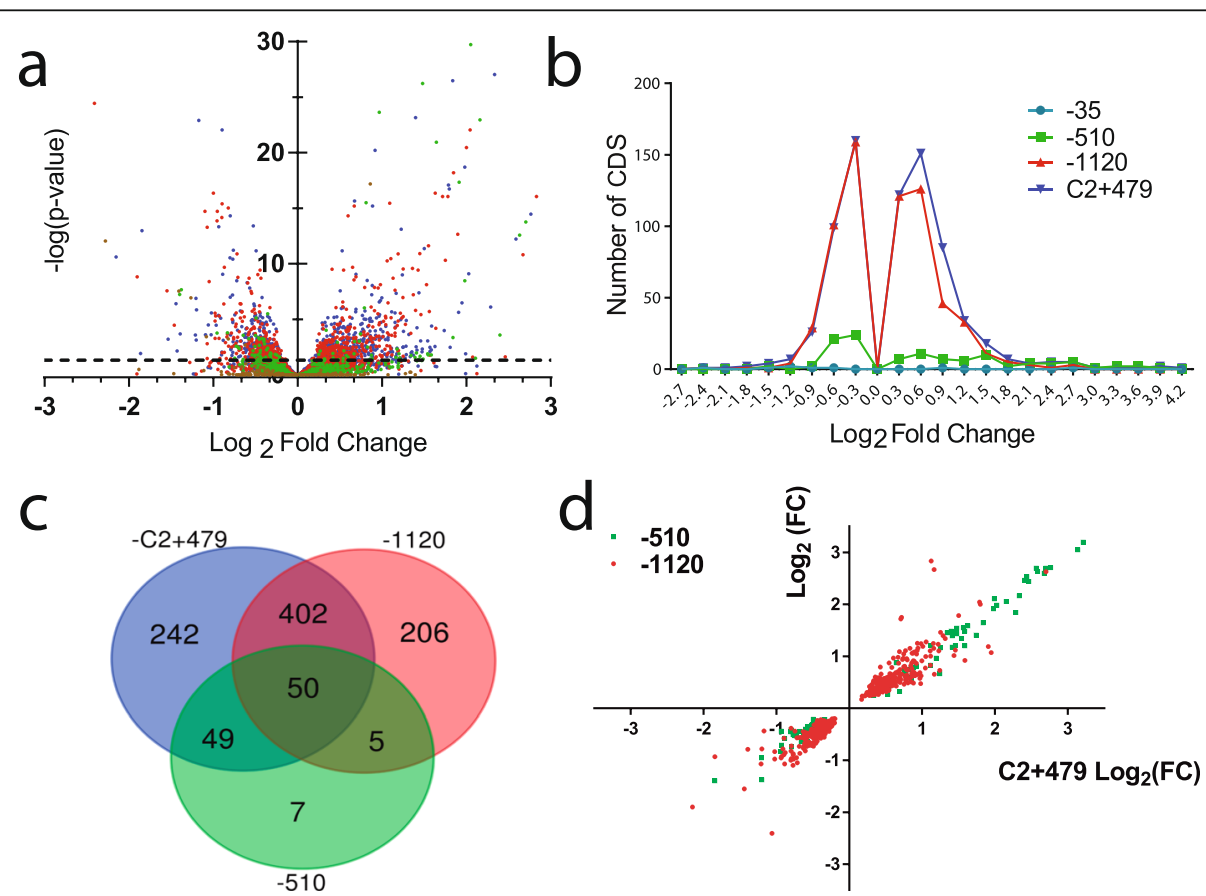

Fig. 4 S10 relocation impacts gene expression genome-wide in a distance-dependent manner. a Volcano plot displaying differentially expressed genes in S10Tnp-35 (brown), S10Tnp-510 (green), S10Tnp-1120 (red), and S10TnpC2+479 (blue). Horizontal dotted line shows $p=0.05$. b The number of coding sequences (CDS) as a function of $\log _{2}(\mathrm{FC}$ ) of strains S10Tnp-35 (turquoise), S10Tnp-510 (green), S10Tnp-1120 (red), and S10TnpC2+479 (blue). c Venn diagram displaying shared genes between S10Tnp-510 (green), S10Tnp-1120 (red), and S10TnpC2+479 (blue). d Expression correlation between movant strains. Dots correspond to individual CDS. The Log $(\mathrm{FC})$ of each gene in S10Tnp-510 (green) or S10Tnp1120 (red) was plotted as a function of $\log _{2}(\mathrm{FC})$ in S10TnpC2+479 
Table 1 Quantitative and qualitative expression changes in the movant strains

\begin{tabular}{lllll}
\hline & -35 & -510 & -1120 & $C 2+479$ \\
\hline Number of upregulated genes & $2(1)$ & $62(37)$ & $361(64)$ & $439(88)$ \\
Mean upregulation $^{\mathrm{a}}$ & $\mathrm{n} / \mathrm{d}$ & $1.5 \pm 0.97$ & $0.67 \pm 0.41$ & $0.78 \pm 0.56$ \\
Number of downregulated genes $_{\text {Mean downregulation }}^{\mathrm{a}}$ & $6(4)$ & $49(2)$ & $301(9)$ & $303(17)$ \\
Total number of altered genes $_{\text {Altered functions }}$ & $\mathrm{n} / \mathrm{d}$ & $-0.5 \pm 0.24$ & $-0.49 \pm 0.26$ & $-0.52 \pm 0.29$ \\
\hline
\end{tabular}

The number of differentially expressed genes $(p<0.05)$ compared to parental strain in fast-growing conditions. The number in parenthesis represents genes whose expression varies more than 2-fold. The magnitude of expression change is quantified as the average of the Log $2(F C) \pm s t a n d a r d ~ d e v i a t i o n$

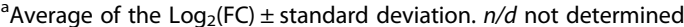

(Fig. 1c), showed 111 genes with significantly altered transcription (Table 1, Fig. 4a, Additional file 2: Data Set S1). Finally, the most affected movants, S10Tnp-1120 and S10TnpC2+479, displayed a transcriptional change in 664 and 742 genes. This represents $17.95 \%$ and $20.06 \%$ of their full gene repertoire. Most of altered genes in the movants were upregulated (Fig. $4 \mathrm{~b}$ and Table 1). These transcriptional perturbations were relatively small in magnitude since only a $26 \%$, a $10.8 \%$, and a $14.15 \%$ of altered genes presented alterations greater than 2-fold in S10Tnp-510, S10Tnp-1120, and S10TnpC2+479, respectively. Meanwhile, upregulated genes showed 2.8-fold, 1.6-fold, and 1.7-fold average increases, respectively (Table 1, Fig. 4b, and Additional file 2: Data Set S1). In the three movants, the downregulated genes displayed a smaller perturbation of $\sim 0.7$-fold (Table 1 ).

The differentially expressed gene profile is similar in S10Tnp-510, S10Tnp-1120, and S10TnpC2+479 movants since a large fraction of transcriptionally altered genes in a movant were also regulated in either of the other two movants (Fig. 4c, Additional file 1: Table S5). Shared genes showed similar levels of transcriptional change across the movants (Fig. $4 \mathrm{~d}$ and Additional file 1: Table S5). For example, the degree of change in altered genes of S10Tnp-510 and S10Tnp-1120 was highly correlated $\left(r=0.927, p<10^{-24}\right)$. The differentially expressed genes were not confined to specific chromosome regions nor associated to a specific replicon: S10 relocation produced homogeneously distributed changes in $V$. cholerae gene transcription (Additional file 1: Fig. S6).

To identify the functions or metabolic pathways altered by $\mathrm{S} 10$ relocation, we classified $V$. cholerae genes in 25 functional categories using the eggNOG database v.4.0 [39] (Additional file 1: Supp. Text). We then identified the categories with over- or under-representation of genes with altered transcription levels in S10Tnp-510, S10Tnp-1120, and S10TnpC2+479 with respect to the full repertoire of $V$. cholerae genome (Additional file 1: Table S6, Fig. S7; Additional file 2: Data Sets 2 and 3).

Genes from the category "Translation, ribosomal structure, and biogenesis" (J) were not significantly altered, which is consistent with the results above showing that S10 relocation did not alter the translation capacity (Fig. 2). The category "Amino acid transport and metabolism" (E) was statistically altered in all three movants. The category "Posttranslational modification, protein turnover, chaperones" (O) was the most affected category in S10Tnp-1120 and S10TnpC2+479, since about $65 \%$ of its genes showed higher transcription in the movants (Additional file 1: Table S6, Additional file 2: Data Set S3). The list of upregulated genes within this category was dominated by chaperones and heat-shock proteins. Strikingly, the highest transcriptional changes occurred in the main pathway for cytosolic protein folding [40]: grpE (VC0854), dnaKJ (VC0855-6), and both copies of the groEL-groES system (VC2664-5 and VCA0819-20). Many transcriptionally altered genes were involved in protein export and ion transport, belonging to several significantly perturbed categories (Table 1 and Additional file 2: Data Set S3). Based on the analysis of functional categories, we observed that $V$. cholerae responds to $\mathrm{S} 10$ relocation by altering amino acid synthesis pathways, by increasing the transcription of chaperones and proteases probably to degrade misfolded proteins, and by activating the expression of transporters and permeases.

\section{Cytoplasm is more fluid in the most affected movants}

During exponential growth, ribosomes account for up to $30 \%$ of bacterial dry weight [41]. S10 encodes half of the ribosomal proteins, very highly expressed constituting more than a third of total E. coli proteins [30]. Therefore, it is likely that a reduction in S10 expression results in macromolecular crowding alterations as observed in other systems [42, 43]. Macromolecular crowding is crucially important in biochemical reactions; however, how it impacts cellular physiology remains mostly unexplored [26-28]. It is well documented that it influences protein folding and aggregation and perturbs protein-nucleic acid interactions [44]. On the other hand, DNA replication has an absolute dependence on macromolecular crowding $[43,45]$. Therefore, the reduction in replication fork dynamics (Fig. 3b, c) and the alteration of 
genes linked to protein folding, protein degradation, permeases, and transport systems (Table 1 and Additional file 2: Data Set S3) observed upon S10 relocation can be interpreted in light of changes in macromolecular crowding caused by a lower RP concentration.

To test this hypothesis, we measured the viscosity of the cytoplasm in the parental strain and in the most affected movants, S10Tnp-1120 and S10TnpC2+479. We expected a more viscous cytoplasm in the parental strain since it expresses S10 genes at higher levels generating a greater concentration of RPs than the movant strains. Differences in cytoplasm viscosity can be uncovered by FRAP experiments on GFP expressing strains. For this, the fluorescence recovery time is measured after bleaching a part of the bacterial cytoplasm [46, 47]. Since the small size and the comma shape of $V$. cholerae complicate the procedure, we generated elongated cells by deleting the Chr2 replication-triggering site (crtS) [25] in cells expressing GFP (Additional file 1: Table S1). These mutants present a defective replication of the secondary chromosome. Therefore, S10TnpC2+479 should have even less copies of S10 per cell and, concomitantly, display higher cytoplasmic fluidity than S10Tnp-1120. The elongated phenotype allows photobleaching part of the cytoplasm.

In the $\Delta c r t S$ context, the parental strain displayed a significantly longer half-time recovery of fluorescence $(\tau)$ than the movants (Fig. 5a, Additional file 1: Supp. Text). The collected data showed a high dispersion due to biological variability; however, $\mathrm{\tau}$ distribution was different in the movants when compared to the parental strain (Fig. 5b) which displayed a $\mathrm{\tau}$ of $139.7 \mathrm{~ms}$ (95\% confidence interval $(\mathrm{CI}) \quad 120.4-158.9 \mathrm{~ms}$; median $=110 \mathrm{~ms}$; $n=104)$. As expected, S10Tnp-1120 showed a $\tau$ of 97.3 ms $(95 \%$ CI $88.31-106.3 \mathrm{~ms} ;$ median $=90 \mathrm{~ms} ; n=128)$, significantly shorter than the parental strain $(p<0.0001)$. S10TnpC2+479 displayed a $\tau$ of $107.5 \mathrm{~ms}(95 \% \mathrm{CI}$ 97.39-117.52 ms; median $=100 \mathrm{~ms} ; n=92)$, statistically lower than the parental strain $(p<0.05)$ but not significantly different from S10Tnp-1120. The more fluid cytoplasm in the movants could be a consequence of fewer S10-encoded RP suggesting that S10 relocation far from ori1 reduces cytoplasm macromolecular crowding.

\section{Growth rate and replication dynamics alterations in movants are alleviated in hyperosmotic conditions}

In line with lower macromolecular crowding, we observed a reduction in cytoplasm viscosity in the movants. To test the possible impact of such molecular crowding alterations on the physiology of the movants, we employed an osmotic stress approach [48-50]. This consists of subjecting strains to a hyperosmotic environment. In these culture conditions, water exits the cell reducing the macromolecular crowding differences between the strains. Therefore, growth differences between the parental strain and the movants should be reduced with increasing solute concentration. To test this, we performed automated growth curves in rich media with increasing $\mathrm{NaCl}$ concentrations, comparing the $\mu$ of the parental strain to S10Tnp-1120 and S10TnpC2+479 movants. As depicted in Fig. 6a, growth rate differences between the parental strain and the movants were reduced as $\mathrm{NaCl}$ concentration increased. Since this phenomenon could be explained by the nature of the solute of choice (e.g., putative differential sensitivity to $\mathrm{NaCl}$ ), we repeated these assays using sucrose as an alternative compound. As shown in Fig. 6b, results were
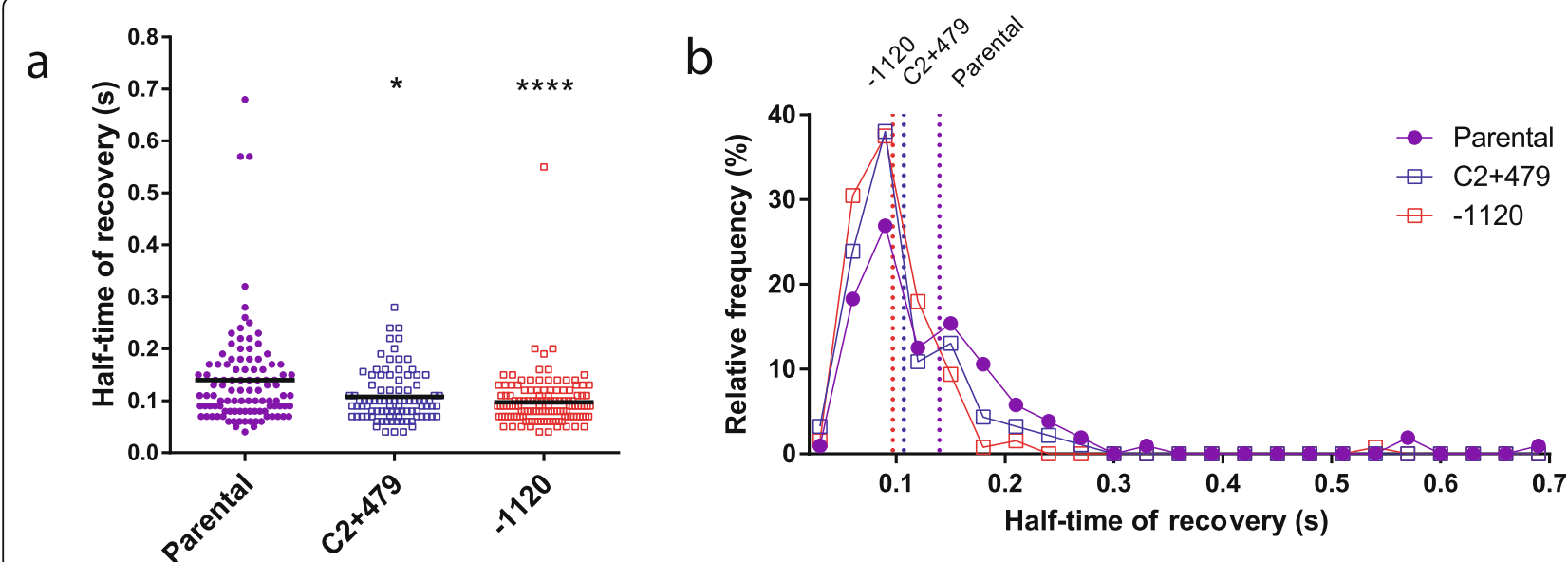

Fig. 5 S10 relocation impacts cytoplasm fluidity. a Half-time of fluorescence recovery $(\tau)$ in the parental-1120 (purple, $n=104)$ and the most affected movants S10Tnp-1120 (red, $n=128$ ) and S10TnpC2+479 (blue, $n=92$ ) in a gfpmut3* $\Delta$ crtS genetic context after bleaching part of the cytoplasm. The line indicates the mean $\tau$ value, and each dot indicates the obtained value for a cell. Statistical significance was analyzed using the Kruskal-Wallis non-parametric tests followed by Dunn's multiple comparisons using parental as control respectively. ${ }^{*} p<0.05$; ${ }^{* * *} p<0.0001$. $\mathbf{b}$ Histogram showing the relative frequency of $\mathrm{\tau}$ to observe the distribution of the values. The vertical dotted line shows the mean value as in a 


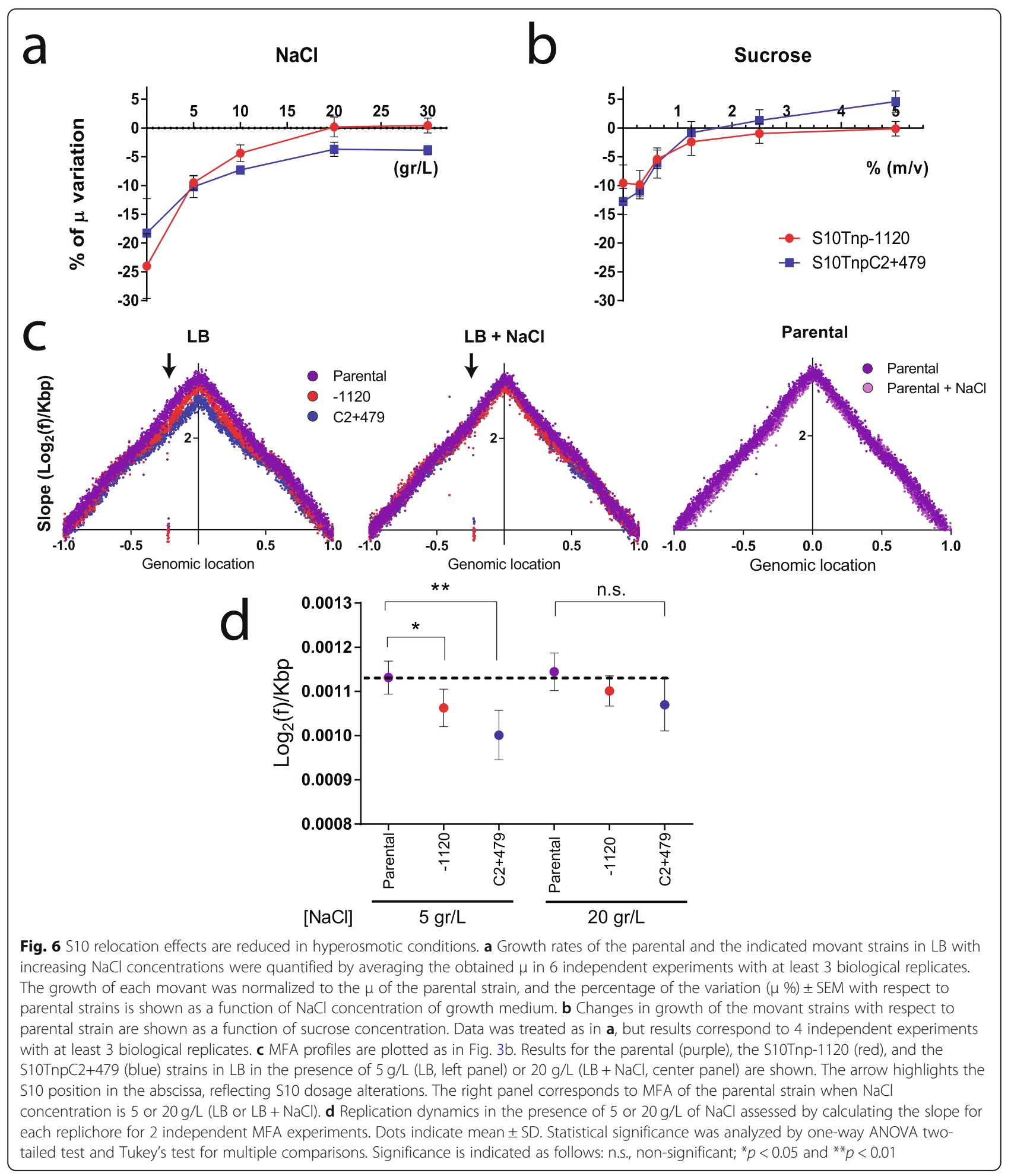

very similar, suggesting that this phenomenon depends on osmotic changes and cannot be attributed to the nature of the solute (see also Additional file 1: Fig. S9).

Notably, the $\mu$ of the parental strain was not significantly reduced in the range of 5 to $20 \mathrm{~g} / \mathrm{L} \mathrm{NaCl}$ (Additional file 1: Fig. S8). Meanwhile, the growth of movants varied significantly along this concentration range, displaying a reduced growth at $5 \mathrm{~g} / \mathrm{L}$ and $10 \mathrm{~g} / \mathrm{L}$ and reaching its maximum at $20 \mathrm{~g} / \mathrm{L}$ (Additional file 1 : Fig. S8). Consequently, growth differences observed are not due to impairment of the parental strain in hyperosmotic conditions. Beyond this concentration growth rate 
is impaired in all strains, probably due to hyperosmotic stress (Additional file 1: Fig. S8, $30 \mathrm{~g} / \mathrm{L}$ ). We conclude that $\mu$ differences caused by S10 relocation far from ori1 can be counterbalanced by artificially increasing cytoplasmic crowding.

Upon S10 relocation far from ori1, we observed a lower replication coverage in the movants suggesting that DNA replication activity diminished, suggesting a lower replication speed in the movants (Fig. 3c). Since molecular crowding is crucial for chromosome replication $[43,45]$, we used the osmotic stress approach to test if the observed replication dynamics defects in movants could be compensated. For this, we performed MFA analyses of the parental strain and the S10Tnp-1120 and S10TnpC2 +479 movants in the presence of 5 or $20 \mathrm{~g} / \mathrm{L}$ of $\mathrm{NaCl}$. In these culture conditions, the parental $\mu$ is unaffected. In contrast, movant strains grew $\sim 12 \%$ slower than the parental strain but they were able to rescue the growth defect at higher $\mathrm{NaCl}$ concentrations (Fig. 6a and Additional file 1: Fig. S8). Importantly, a concentration of $20 \mathrm{~g} / \mathrm{L}$ of this solute increased external osmolality without impacting general physiology. We avoided higher $\mathrm{NaCl}$ concentrations that could lead to pleiotropic effects (Additional file 1: Fig. S8). Indeed, the addition of the solute had no effect on the replication dynamics of the parental strain (Fig. 6c, right panel, and 6d). As in earlier experiments, MFA analyses revealed that the movants have a significantly lower slope than the parental strain. Increasing $\mathrm{NaCl}$ concentration to 20 $\mathrm{g} / \mathrm{L}$ made their slopes converge diminishing replication dynamics differences (Fig. 6c, d, Additional file 1: Fig. S10). The integration of these and the previous observations suggests that lower expression of RP caused by S10 relocation (Fig. 1b) leads to lower macromolecular crowding (Fig. 5), which negatively impacts replication (Fig. 3b). This fits the observation that addition of external $\mathrm{NaCl}$, causing water loss and thus narrowing differences in macromolecular crowding, leads to similar replication dynamics between the parental and the movant strains (Fig. 6d, Additional file 1: Fig. S10).

\section{Discussion}

Comparative genomics suggests that gene order coordinates cell cycle to the expression of key functions necessary for cellular homeostasis $[4,11,19,20]$, but few papers provided experimental support [13, 14, 51]. A notable case is that of ribosomal genes which are located near the oriC in fast-growing bacteria $[19,20]$. By systematically relocating $\mathrm{S} 10$, the main cluster of RP genes (Fig. 1c), we proved that its genomic location determines its dosage and expression in $V$. cholerae (Fig. 1b). S10 repositioning far from ori1 leads to larger generation times, lower fitness, and less infectivity [22, 23]. These effects are dependent on S10 dosage. However, the mechanism explaining how RP dosage affects cell physiology was still missing.

The most straightforward explanation was that high RP dosage due to multifork replication increases their expression maximizing protein biosynthesis capacity [19, 20]. Our initial hypothesis was that movants in which S10 was far from ori1 would have a lower translation capacity, easily explaining lower growth and fitness of these movants. Surprisingly, we found that in the most affected movants, translation capacity reduction could not explain the observed physiological changes (Fig. 2). We do not rule out that translation impairment may have an effect in the cellular physiology; however, it must have a secondary role in the phenotypes displayed in the affected movants. Slight differences in protein production between the parental strain and the most affected movants could only be detected when measuring maximum protein synthesis capacity (Additional file 1 : Fig. S1). All strains showed similar sensitivity to ribosome-targeted antibiotics, suggesting similar ribosome numbers (Fig. 2c). The movants displayed a larger proportion of assembled ribosomal subunits; hence, more free ribosomal subunits are engaged in translation in the movant strain, compensating putative deficiencies in the translation apparatus (Fig. 2e). Interestingly, the S10TnpC2+479 displayed a small peak of $\sim 21 \mathrm{~s}$ that might correspond to precursors of 30s subunit typically associated to cells displaying ribosome assembly deficiencies [52]. Meanwhile, complementation of movants with $\sec Y$ and rpoA, two S10 genes not related to ribosome biogenesis, failed to rescue the growth defect demonstrating the relevance of RP in the observed phenotype. In sum, although dosage reduction of S10encoded RP genes caused the observed phenotypes, it is unlikely that this is a consequence of translation defects.

Deep sequencing techniques revealed less transcriptional activity in the region flanking ori1 (Fig. 3a) and lower replication velocity in the most affected movants (Figs. 3b, c and 6c). Since highly expressed genes that account for a large majority of transcriptional activity in the cell (i.e., rrn, RP genes) cluster at this chromosomal region, slight changes in its dosage may globally impact cell physiology $[4,11]$ and may be responsible for the slight reduction in translational activity observed (Additional file 1: Fig. S1). Meanwhile, differential expression analysis revealed that the transcriptional response is not limited to the ori1 region (Fig. S6), and encompasses a large number of genes that show slightly but consistently altered transcription in the most affected movants (Fig. 4). Furthermore, the number of these genes increases with distance between S10 and ori1 (Table 1, Fig. $4 \mathrm{a}, \mathrm{b}$ and S6). The latter observation corresponds to biologically meaningful transcriptional changes since furthest relocations caused larger perturbations (Fig. 4a, 
b), and the majority of altered genes were common to the different movants (Fig. 4c), where they showed similar transcriptional changes (Fig. 4d). This strongly suggests the presence of a common mechanism that slightly affects gene expression at a large scale. Amino acid metabolism and transport genes were less transcribed while there was an upregulation of genes helping protein folding and cellular transporters (Table S5, Additional file 2: Data sets S1 and S3). Importantly, and in line with previous data (Fig. 2), the transcription of translation genes seems to be unaffected in the movants reinforcing the notion that lower protein biosynthesis capacity was not enough to explain the physiological alterations that we observed.

Molecular crowding has a well-known key role in biochemical reactions. Even if its impact on physiological processes has been poorly studied [28], two processesDNA replication and protein folding-are strongly influenced by macromolecular crowding [29]. Since the discovery of DNA replication, the presence of crowding agents such as polyethylene glycol was shown to be absolutely necessary to reproduce DNA polymerase activity in vitro $[43,45]$. In parallel, macromolecular crowding greatly impacts protein aggregation and folding [29], although the in vivo consequences of how the latter occurs are still a matter of debate [44,53]. It was recently shown that ribosomes are important contributors of macromolecular crowding in the cytoplasm both in prokaryotic and eukaryotic systems [42, 43]. All this information leads us to suggest that upon S10 relocation, the consequent fewer RP may lead to homeocrowding [26] perturbations. To the best of our knowledge, this is the first study exploring the consequences of lower macromolecular crowding conditions since most works linking this physicochemical factor to physiology focus on situations of increased crowding $[43,54,55]$. Concomitantly, we observed reduced replication activity (Fig. 3c), as well as induction of proteases and chaperones to cope with protein aggregation and misfolding (Table 1 and Additional file 1: Fig. S6). Notably, in the most affected movants, the genes coding for the three main chaperone systems-grpE, dnaKJ, and groEL-groES [40]-were among the most strongly induced. The lower transcription of protein and ion transporters could be used for intracellular environment restoration (Table S4, Additional file 1: Fig. S6) and could be a natural consequence of the change in cytoplasm osmotic pressure. We next tested experimentally if S10 relocation could alter homeocrowding. First, using FRAP, we observed slight but statistically significant alterations in the fluidity of the cytoplasm of the most affected movants compared to the parental strain (Fig. 5 a, b, Additional file 1: Supplementary Text). This supports the notion that lower expression of RP associated with movants lowers cytoplasm macromolecular crowding. In the $\Delta c r t S$ context, we did not detect differences in cytoplasmic fluidity between the S10Tnp-1120 and S10TnpC2+479 movants, expected from lower S10 copy number in the latter by Chr2 loss. We believe that the detrimental effects of $c r t S$ deletion [25] can explain this. In the S10TnpC2+479 movant, S10 dosage reduction enhances fitness loss, as reflected by slower growth and the presence of small non-viable cells in the microscope not further analyzed (data not shown). When Chr2 replication is inhibited, the fusion of both chromosomes-mainly between their terminal regions-occurs at relatively high frequency [56]. Therefore, the S10TnpC2+479 $\Delta$ crtS population might in part consist of cells with fused chromosomes. In this scenario, S10 dosage would not decrease below 1 copy per cell.

The osmotic stress approach provided strong evidence supporting the notion that S10 dosage deficit perturbs cellular homeocrowding. In rich medium, movant strains grow slower than the parental strain. With increasing solute concentrations, this growth deficit is reduced (Fig. 6a, b). In the case of $\mathrm{NaCl}$, the parental strain grew normally in the range from 5 to $20 \mathrm{~g} / \mathrm{L}$ (Additional file 1 : Fig. S8). Outside of this range, growth rate was reduced. Growth was particularly impaired at concentrations below $5 \mathrm{~g} / \mathrm{L}$ where culture development was very variable due to hyposmotic stress (Fig. 6a and data not shown). Interestingly, movants looked more sensitive than the parental strain to lower solute concentrations. We think that movants express less ribosomal proteins which account for a large fraction of the bacterial proteome, which in turn constitutes a large proportion of the cytoplasmic macromolecules [57]. It is known that about $0.5 \mathrm{~g}$ of water is bound per gram of cytoplasmic macromolecules $[48,58]$. Therefore, movants may lose their capacity to retain water, suffering from a situation similar to being exposed to hyposmotic conditions. Meanwhile, the $\mu$ of the parental and the movants was similar when exposed to $20 \mathrm{~g} / \mathrm{L}$ or beyond. All strains suffered from physiological alteration beyond this concentration since at $30 \mathrm{~g} / \mathrm{L}$ of $\mathrm{NaCl}$ they displayed a growth impairment compared to the $20 \mathrm{~g} / \mathrm{L}$, suggesting that detrimental hyperosmotic conditions altered the strains similarly.

Recent work shows that specific ribosomal protein genes link cell growth to replication in Bacillus subtilis [59]. We observed similar effects since S10 dosage correlated growth rate and oriC-firing frequency (Fig. 3b, c, S6 and Table S3). In the cited study, the authors attribute this effect to ribosomal function. Although in our system, the effects were milder, we do not rule out the possibility that S10 relocation alters cellular physiology through a reduction in protein synthesis. But this effect is unlikely to account for the full magnitude of the observed phenotypes (Fig. 2) especially as it is relieved in 
hyperosmotic conditions. We believe that this could be due to a number of factors including the following: (i) the many regulatory mechanisms that control ribosomal protein expression at the translation level, which could partially compensate transcription reduction; (ii) the fact that ribosomal subunits are found in excess with respect to assembled ribosomes; (iii) the possibility that an eventual reduction in functional ribosomes can be compensated by faster translation rates [60-62]; and (iv) finally, it has been described, particularly in Vibrio sp. CCUG 15956 [63], that ribosomes are available in excess of numbers needed for exponential growth. Such large ribosome quantities would have been selected as an ecological survival strategy that allows for fast growth restoration after its arrest in rapidly changing environmental conditions [64]. Hence, lower S10 expression could be buffered at many levels and protein production might be only mildly impacted. Molecular crowding reduction might however not be as easily compensated. Therefore, movant strains possess a less crowded cytoplasm where DNA polymerase activity is reduced and more chaperones are needed. This would embody a novel mechanism which could explain how ribosomal protein gene position influences growth rate.

\section{Conclusions}

The order of key genes along the chromosomal ori-ter axis is phylogenetically conserved in bacteria [11]. However, its influence in cell physiology and its role in genome evolution remain unclear. The chromosomal position of RP genes is baised towards oriC, particularly in fast-growing bacteria [20]. A very reasonable explanation for this observation is that such positional tendency allows recruiting multifork replication to enhance $\mathrm{RP}$ expression and ribosome biogenesis during exponential growth. Indeed, bacterial growth closely correlates to ribosomal protein content. This has been attributed to the role ribosomes have in protein synthesis $[65,66]$. We propose that on top of that, ribosome concentration may change the macromolecular crowding conditions to optimize biochemical reactions, in particular in protein folding and DNA replication [28, 29]. We provide evidence indicating that this is the case for replication dynamics in $V$. cholerae. Our experiments suggest that the genomic position of S10 contributes to generate the RP levels necessary to attain optimal cytoplasmic macromolecular crowding. Besides connecting ribosomal gene position to growth in $V$. cholerae, this mechanism could link ribosome biogenesis to cell cycle in bacteria. During exponential phase, when RP production is maximal and ribosomes represent $30 \%$ of cell weight, crowding peaks. This leads to the highest oriC-firing frequency. Upon nutrient exhaustion, ribosome production is reduced and the cytoplasm macromolecular crowding diminishes, slowing down replisome dynamics.

\section{Materials and methods General procedures}

Genomic DNA was extracted using the GeneJET Genomic DNA Purification Kit while plasmid DNA was extracted using the GeneJET Plasmid Miniprep Kit (Thermo Scientific). PCR assays were performed using Phusion High-Fidelity PCR Master Mix (Thermo Scientific). Strains and plasmids used in this study are listed in Table S1.

\section{Culture conditions}

For fast-growing conditions, bacterial cultures were done in Lysogeny Broth Lennox formulation (LB) at $37^{\circ} \mathrm{C}$ with maximum agitation. For harvesting cells in fast-growing conditions, $\sim 30 \mu \mathrm{L}$ of an $\mathrm{ON}$ culture was used to inoculate pre-warmed $250 \mathrm{~mL}$ Erlenmeyer flasks with $70 \mathrm{~mL}$ of LB and agitation was set to $250 \mathrm{rpm}$. For selection, the following antibiotic concentrations were used: chloramphenicol $(3 \mu \mathrm{g} / \mathrm{mL})$, kanamycin $(25 \mu \mathrm{g} / \mathrm{mL})$, spectinomycin $(100 \mu \mathrm{g} /$ $\mathrm{mL})$, carbenicillin $(50 \mu \mathrm{g} / \mathrm{mL})$, and zeocin $(25 \mu \mathrm{g} / \mathrm{mL})$. NaCl and sucrose were added at the indicated concentrations. Strains and plasmids used are listed in Table S1. For strains expressing $\sec Y$ and rpoA from pBAD43, cells were cultured in LB (for leak expression), in LB supplemented with $1 \%$ glucose (expression repression), or in LB with $0.2 \%$ arabinose (maximum induction).

\section{Automated growth curve measurements}

Automated growth curves were performed in 96-well plates avoiding the use of external rows and columns. ON cultures were diluted 1/1000 in LB. Bacterial preparations were distributed at least by triplicate in p96 microplates. Growth curve experiments were performed using a TECAN Infinite Sunrise microplate reader (Thermo), following the $\mathrm{OD}_{600 \mathrm{~nm}}$ every $5 \mathrm{~min}$ at $37^{\circ} \mathrm{C}$ on maximum agitation. Growth rate was obtained using a custom Python script coupled to the Growthrates program [67].

\section{Protein production capacity}

For estimating GFP production, we performed V. cholerae gfpmut $3^{*}$ automated growth curves in a TECAN Infinite 200 microplate reader (Thermo), following $\mathrm{OD}_{600 \mathrm{~nm}}$ and GFP fluorescence over time. Data was analyzed using GraphPad Prism 6. For flow cytometry, strains were grown in fast-growing conditions until early exponential phase $\left(\mathrm{OD}_{450} \sim 0.2\right)$. Then, $50 \mu \mathrm{L}$ was diluted in $800 \mu \mathrm{L}$ of PBS. The fluorescence of 20.000 events was recorded in a MACSQuant 10 analyzer (Miltenyi Biotec). Cells were detected using Side Scatter Chanel (SSC) in $\log _{10}$ scale. Data analysis was done using the Flowing 
Software 2.5.1 (www.flowingsoftware.com). For luciferase activity measurement, Vibrio cholerae::RL strains were cultured until $\mathrm{OD}_{450 \mathrm{~nm}} \sim 0.2$. For each experiment, three samples of $20 \mu \mathrm{L}$ were harvested and directly measured using the Renilla Luciferase Assay System (Promega).

\section{MIC determination}

The MICs of Gm, Cm, and Er were determined using Etest $^{\circ}$ and the disk diffusion method following the manufacturer's instructions (Biomérieux).

\section{Ribosome profiling}

Ribosomal 70s, 50s, and 30s species from the indicated $V$. cholerae strains were isolated as previously described $[68,69]$. Early exponential phase cultures $\left(\mathrm{OD}_{450 \mathrm{~nm}} \sim\right.$ 0.2 ) were harvested by centrifugation. Subsequent steps were performed at $4{ }^{\circ} \mathrm{C}$. The pellet was resuspended in ice-cold buffer A (20 mM HEPES pH 7.5, $50 \mathrm{mM} \mathrm{NH4Cl}$, $10 \mathrm{mM} \quad \mathrm{MgCl} 2, \quad 5 \mathrm{mM} \quad \beta$-mercaptoethanol, $0.1 \mathrm{mM}$ PMSF) in the presence of Ribolock (Thermo Fisher Scientific). DNase I was added up to $2 \mu \mathrm{g} / \mathrm{mL}$ and kept for $20 \mathrm{~min}$ at $4{ }^{\circ} \mathrm{C}$. Cells were lysed by two passes at $11,000-$ 15,000 psi using Emulsiflex. Cell debris were removed by two centrifugation steps at $30,000 \mathrm{~g}$ for $30 \mathrm{~min}$. Then, $0.8 \mathrm{~mL}$ of cold $60 \%$ sucrose buffer $\mathrm{A}$ was added to RNAse-free $5 \mathrm{~mL}$ ultraclean tubes for ultracentrifugation in a SW55Ti (Beckman). The ribosome-containing supernatant was used to fill these tubes, and an ultracentrifugation step was performed for $16 \mathrm{~h}$ at $150,000 \mathrm{~g}$. Ribosomes were recovered from the bottom $0.8 \mathrm{~mL}$ of $60 \%$ sucrose buffer A and dialyzed using a Float-a-lyzer G2 in buffer A. Sedimentation velocity was determined in a Beckman XL-I Analytical Ultracentrifuge. Double sector quartz cells were loaded with $400 \mu \mathrm{L}$ of buffer A as reference and $380 \mu \mathrm{L}$ of sample $(3 \mu \mathrm{m})$, and data were collected at $120,000 \mathrm{rpm}$ from 5.8 to $7.3 \mathrm{~cm}$ using a step size of $0.003 \mathrm{~cm}$ without averaging. Sedimentation velocity data were analyzed using the continuous sizedistribution model employing the program SEDFIT.

\section{RNA preparation and sequencing for transcriptomic studies}

RNA was prepared as described in [70]. We performed four independent biological replicates for each sample. Briefly, $20 \mathrm{~mL}$ of an early exponential phase culture was recovered by centrifugation at $4500 \mathrm{rpm}$ for $10 \mathrm{~min}$ at $4{ }^{\circ} \mathrm{C}$. Then, RNA was extracted using TRIzol (Thermo Fisher Scientific). Residual DNA was removed with TURBO DNAse (Ambion). RNA quality (total, depleted, and purified) was checked on the Bioanalyzer 2100 (Agilent). Samples were checked for RNA integrity number $>$ 8. The rRNA was depleted using the MicrobExpress kit (Ambion), and libraries were built using the TruSeq Stranded RNA LT Sample Prep Kit (Illumina) and checked for concentration and quality on Bioanalyzer and QuBit (Invitrogen). Sequencing of multiplexed libraries was performed on a HiSeq 2500 (Illumina). Then, in-house quality control process was applied to reads that passed the Illumina quality filters (raw reads). The sequences of the Illumina adapters and primers used during the library construction were removed from the whole reads. Low-quality nucleotides were removed from both ends. Trimmed reads were aligned to the $V$. cholerae reference genome using Bowtie [71] with default parameters. Aligned reads were counted using HTSeq Count [72]. Further quality control and differential expression analysis was performed using methods described above [73-75]. Graphics were done using the GraphPad software, specific online service for Venn diagram (http://bioinformatics.psb.ugent.be/webtools/ Venn/) and Circos Plot [76]. The sequence data was submitted to the GenBank Sequence Read Archive (SRA) (see above). Accession numbers for these samples are SRR8316520, SRR8316521, SRR8316528, SRR8316529, SRR8316526, SRR8316527, SRR8316524, SRR8316525, SRR8316522, SRR8316523, SRR8316530, SRR8316531, SRR8316518, SRR8316519, SRR8316516, SRR8316517, SRR8316514, SRR8316515, SRR8316512, and SRR8316513.

\section{RNA-seq statistical analysis}

Count data were analyzed using $\mathrm{R}$ version 3.1.2 [77] and the Bioconductor package DESeq2 version 1.6.1 [73]. Data were normalized with DESeq 2 and the "shorth" parameter. The dispersion estimation and statistical test for differential expression were performed with default parameters (including outlier detection and independent filtering). The generalized linear model was set with strain (parental, S10Tnp-1120, S10Tnp-35, S10Tnp-510, and S10TnpC2+479 levels) as main effect. Since samples were prepared 4 times independently, the date of sample preparation was also included into the model as a blocking factor to catch more variability and increase the statistical power. Raw $p$ values were adjusted for multiple testing according to the Benjamini and Hochsberg $(\mathrm{BH})$ procedure [74], and genes with an adjusted $p$ value lower than 0.05 were considered differentially expressed.

\section{Whole chromosome transcriptional activity comparisons}

Reads were mapped as previously described [78] to a custom assembled linear version of the $V$. cholerae that starts (base 0) at the ter and finishes at the ter, with the oril at the center of the sequence. Total reads mapped to this sequence were counted and normalized as previously described [78]. Fold changes were calculated using normalized values, and $p$ values were calculated as previously described [78]. 


\section{Marker frequency analysis, slope, and ori1/ter 1 ratio calculation}

Genomic DNA extracted from early exponential phase $\left(\mathrm{OD}_{450 \mathrm{~nm}} \sim 0.15\right)$ was used for library preparation using a PCR-free protocol. Libraries were sequenced on an Illumina MiSeq sequencer using 100- to 150-base-length paired-end reads for $100 \times$ genome coverage. The resulting trimmed FastQ files were analyzed using R2R script to obtain the frequency of each locus along the genome, removing repeated sequences $[23,25,36]$. Then, the $\log _{2}$ frequencies every 1000 -bp window were then plotted as a function of their relative position on chromosome 1 in ter1-ori1-ter1 order. Slopes were obtained from linear regression of plots of $\log _{2}$ frequencies along replichore length from ter1 to ori1 $\left(R^{2}>0.95\right)$. The slopes represent the $\log _{2}$ frequency change per kilobase pair. The frequency of ori1 and ter 1 was quantified by averaging 50 frequency data points corresponding to ori1 and ter1 zones. The S10 frequency was calculated by averaging panels corresponding to VC2569 and VC2599, respectively. These values were used to calculate S10 dosage by calculating the S10/ter1 ratio. The sequence data was submitted to GenBank SRA under the following accession numbers: SRR11398735, SRR11398734, SRR11398726, SRR11398731, SRR11398733, SRR11398727, SRR11398728, SRR11398732, SRR11398729, SRR11398730, SRR11396016, SRR11396017, SRR11396015, SRR11396018, SRR11396019, SRR11396014, SRR11396020, SRR11393293, SRR11393294, SRR11392757, SRR11392758, SRR11392762, SRR11392760, SRR11392754, SRR11392756, SRR11392755, SRR11392763, SRR11392752, SRR11392753, SRR11392759, SRR11392761, SRR11365216, SRR11365215, SRR11365217, SRR11365218, SRR11365214, SRR11365132, SRR11365130, SRR11365133, SRR11365131, SRR11365134, and SRR11363959.

\section{Functional characterization of the transcriptomic response}

$V$. cholerae N16961 genes were aligned against the eggNOG database v.4.0 [39]. Only hits with at least 50\% similarity and $e$ value $<0.05$ were used. Each protein was assigned to the best functional category, according to the percentage of similarity and the length of the alignment. We then calculated the fraction of categories enriched in the fraction of differentially expressed genes, compared to abundances of the different eggNOG categories in the $V$. cholerae genome. The over- or underrepresentation of protein families was assessed statistically using the Pearson chi-square test with the Benjamini-Hochberg correction for multiple test. For further validation, this test was performed 10,000 times in random subsamples of $30 \%$ of the differentially expressed genes.

\section{FRAP}

For measurement of GFP synthesis, stationary phase cultures of $V$. cholerae strains were diluted $1 / 300$ in fresh LB. Then, $6 \mu \mathrm{L}$ was distributed on an LB agar pad within a Gene Frame (Thermo Fisher) and covered with a cover slip. When indicated, the agar pad was supplemented with $\mathrm{Cm}$ at MIC. Cells were then visualized and recorded in a Spinning-Disk UltraView VOX (PerkinElmer) equipped with two Hamamatsu EM-CCD (ImageEM X2) cameras. Photobleaching was done using $5-20 \%$ of laser power.

For long-term experiments (GFP re-synthesis), detection images were taken every $2 \mathrm{~s}$ after photobleaching the total area of the cell for at least 5 min using 200$500 \mathrm{~ms}$ of acquisition time. Image analysis was done using Image following photobleached and non-bleached cells in time. The average signal of not-photobleached cells was subtracted to the signal of bleached cells to take into account the decay produced by cell imaging.

For measurement of GFP diffusion within bacteria, we used the exposure times $20 \mathrm{~ms}$ at maximum acquisition frame rate after photobleaching a part of the cell area. Then, these movies were analyzed using a specific Jython script developed during the Image Processing School Pilsen 2009 and updated to modern Fiji as described (ImageJ: Analyze FRAP movies with a Jython script, https:// imagej.net/Analyze_FRAP_movies_with_a_Jython_script. Accessed 14 August 2019). For every cell analyzed cell length, the photobleached area and the total cell area were determined. Also, a control area was measured. Then, the Jython script was executed. For data analysis, we only kept cells shorter than $6 \mu \mathrm{m}$. We only registered half-time values when the function fitted with $R^{2}>0.8$ (see Additional file 1, Supp. Text, Additional Analysis of FRAP images for further details).

\section{Supplementary information}

Supplementary information accompanies this paper at https://doi.org/10. 1186/s12915-020-00777-5.

\footnotetext{
Additional file 1: Figure S1. The most affected movants, display lower GFP production than the Parental strain at the single cell level. FRAP experiments were performed in $L B$ at $37^{\circ} \mathrm{C}$ taking a photo every 2 seconds for at least 5 minutes using the Parental-1120 strain (Parental, violet), the S10Tnp-1120 (red) and S10TnpC2+479 (blue) movants. The parental was also tested in presence of chloramphenicol at MIC $(+\mathrm{Cm})$. a) A representative plot showing the recovery of fluorescence over time in individual cells. b) The percentage of FRAP at the endpoint of the experiment is shown for all cells tested. Mean with $95 \% \mathrm{Cl}$ is shown. Statistical significance was analyzed by Kruskal-Wallis test $(p<0.0001)$. Then Dunn multiple comparison test was made for mean rank obtained for each strain. Letters denote groups being statistically different. Figure S2. rpoA and secY overexpression does not rescue growth rate impairment due to $\mathrm{S} 10$ relocation. Effect of empty vector, rpoA or secY expression
} 
was quantified by averaging the slope $(\mu)$ obtained using 4 biological replicates for each strain in different induction conditions. Results are expressed as the mean $\mu \pm 95 \% \mathrm{Cl}$. Statistical significance was analyzed using a two-way ANOVA two tailed test and Tukey test for multiple comparisons $(p<0.0001)$. Independently of culture conditions differences are not statistically significant between strains harboring the empty vector, pASB25 or pASB26. Expression was repressed by supplementing culture media with $1 \%$ glucose. Induction was achieved adding $L$-arabinose up to $0.2 \%$. Figure S3. RNA Coverage of Chromosome 1 (Chr1) on the full movant strain set. RNA prepared in exponential phase was DeepSequenced as described in Materials and Methods. Reads were mapped along the Chr1 of $\mathrm{V}$. cholerae and normalized against the full sequence volume. The graphs show the coverage as Normalized Expression Values (dotted lines indicate 75, 50 and $25 \mathrm{e} 10^{3} \mathrm{NEV}$ ) along both replichores of the replicon in ter1-ori7-ter1 order. Each graph represents one strain: Parental (purple); S10Tnp-35 (cyan); S10Tnp-510 (green); S10Tnp-1120 (red); S10TnpC2+479 (blue). The $400 \mathrm{Kbp}$ flanking ori1 are highlighted in orange. A red arrow indicates the peak corresponding to the S10 locus. The coverage of the orit region and the size of the S10 peak lowers with increasing S10-ori1 distance (see Table S3). This was not the case for Chr2 where the transcriptional activity of the ori2 region was similar in all strains. Curiously a small increase of the transcriptional activity of the superintegron [81] was observed in S10Tnp-1120 movant (Fig. S4). Figure S4. RNA Coverage of chromosome 2 (Chr2) on selected strains. RNA prepared in fast-growing conditions was subjected to deep sequencing. Reads were mapped along the Chr2 of $V$. cholerae. The graphs show Normalized Expression Values along both replichores of the replicon in ter2oriz-ter2 order of the parental and the most affected strains. Each graph represents the coverage along Chr2 length of Parental (purple), S10Tnp1120 (red) and S10TnpC2+479 (blue). The superintegron [81] is highlighted in red (SI). Interestingly, SI region is overexpressed in the S10Tnp-1120 movant. Scale from the first base is shown above the graphs. Figure S5. Replication dynamics closely correlates S10 location, dosage, oril firing and growth rate. a) The slopes obtained from the MFA analyses (white circles, right axis) and the growth rate (black squares, left axis) of each strain were plotted as a function of the $\mathbf{S 1 0}$ genomic location. b) S10 dosage (black triangles, left axis) and ori1/ter1 (white triangles, right axis) ratio from MFA analyses for each strain were graphed as a function of the S10 positioning. c) S10 dosage (red), ori1/ter1 ratio (green) and growth rate (blue) are plotted as a function the slope obtained for each strain in MFA analyses. Linear regression for each variable is shown in dotted lines. The data used for each graphic can be found in Table S3. The obtained correlations and their statistical significance are described in the main text of the article. Figure $\mathbf{S 6 . ~ S 1 0 ~ r e l o c a t i o n ~ p r o d u c e s ~ h o m o - ~}$ geneously distributed global changes in $V$. cholerae gene expression. Circos plot of genome-wide expression data from strains S10Tnp-35 (Turquoise), S10Tnp-510 (green), S10Tnp-1120 (red) and S10TnpC2+479 (blue). Upper case represents Chr1 while lower case is Chr2 in ter-ori-ter disposition. The origin of replication of each chromosome is represented as oriC1 and oric2 respectively. From inside to outside: Sense and antisense $\mathrm{V}$. cholerae genes are depicted as dark orange and orange boxes, respectively. Blue bars represent RNA-seq read counts per gene (scale 1200,000). Fold-change expression relative to the parental strain is indicated as a green or red solid line indicating fold-expression differences higher than 1.2 or lower than 0.8 , respetcively. Dark red dots indicate $\log (p$-value) of the differential expression analysis. Notably, the abundance of significantly altered genes (red dots) from left to right. Figure

S7. Manhattan Pot showing statistically altered functions across the movant strain set. The abscissa corresponds to specific COG within the S10Tnp-510 (green), S10Tnp-1120 (red) and S10TnpC2+479(blue). S10Tnp35 is not included since very few genes are differentially expressed displaying no altered functions. The purple line indicates statistical significance fixing a in 0.05 . Figure S8. The growth rate of the parental strain, S10Tnp-1120 and S10TnpC2+479 was measured using automated growth curves at different $\mathrm{NaCl}$ concentrations in rich medium. The mean $\mu$ value with SEM of 5 independent experiments is shown. Statistical significance was analyzed by one-way ANOVA two-tailed test. Then Holm-Sidak test was done to compare the means values obtained for each strain. Letters denote groups being statistically different within strains. Differences between strains within each $\mathrm{NaCl}$ concentration are denoted as follows: * $p<0.05 ;{ }^{*}, p<0.01 ;{ }^{* * *}, p<0.001$ and n.s. stands for non-significant. Figure S9. The growth rate of the parental strain, S10Tnp-1120 and S10TnpC2+479 was measured using automated growth curves at different concentrations of sucrose in LB. The mean $\mu$ value with SD of 4 independent experiments by triplicate is shown. All experiments showed the same trend. Statistical significance was analyzed by two-way ANOVA twotailed test. Then Holm-Sidak test was done to compare the means values obtained for each strain. Letters denote groups being statistically different within strains. Differences between strains within each sucrose concentration are denoted as follows: ${ }^{*}, p<0.05 ;{ }^{* * *}, p<0.0001$ and n.s. stands for non-significant. Figure S10. MFA profiles plotted as in Fig. 3b and Fig. $6 c$. Results for the parental strain (purple), S10Tnp-1120 (red) and the S10TnpC2+479 (blue) movants in LB in presence of $5 \mathrm{gr} / \mathrm{L}$ (light) or 20 $\mathrm{gr} / \mathrm{L}$ (dark) are shown. The statistical analysis of these experiments is shown in Figure $6 \mathrm{~d}$. Table S1. Full list of plasmids, bacteria strains used in this study. Table S2. Exponential fit of fluorescence (GFP production) as a function of $\mathrm{OD}_{600 \mathrm{~nm}}$. Data was adjusted to the equation $\mathrm{Y}=$ $Y_{0}^{*} \exp \left(k^{*} X\right)$. Table S3. Quantification of genome wide transcriptional activity. We calculated the read coverage of the $400 \mathrm{Kbp}$ flanking ori1 [78]. Table S4: Slope, S10 dosage and ori1/ter1 ratio obtained from MFA analysis and growth rate of the analyzed strain set. Table S5. Transcriptionally altered genes are shared between movants and regulated in the same manner. The proportion of altered genes that is also found to be regulated in either of the other two movants is shown in the first column (grey). The percentage is shown in parentheses. The two entry table shows that these altered genes were transcriptionally altered in the same way between the movants. We calculated the Pearson Correlation Test for their $\log _{2}(F C)$. The value of the test is shown in green while the corresponding $\mathrm{p}$-value is displayed in orange. Table S6. Altered functions upon $\mathrm{S} 10$ relocation. Genes within $V$. cholerae genome were classified in functional categories using eggNOG database. The table shows the number of genes whose expression is altered in selected functional categories for each movant train and the total genes in the chromosome belonging to each category. The number in parenthesis represents the $\%$ with respect the total number of genes. The functions with no genes or with no alterations are not displayed and can be found in Additional file 2, Data Set S3. The total number in the last row also includes functions not displayed.

Additional file 2. Data sets of differentially expressed genes and classification into functional categories.

\section{Abbreviations}

RP: Ribosomal protein; oriC: Origin of replication; S10: s10-spc-a; ter: Terminal region; Chr1: Main chromosome; Chr2: Secondary chromosome; Kbp: Kilobase pairs; Mbp: Megabase pairs; $\mu$ : Growth rate; OD: Optical density; GFP: Green fluorescent protein; RL: Renilla luciferase; MIC: Minimum inhibitory concentration; Cm: Chloramphenicol; Gm: Gentamicin; Er: Erythromycin; FRAP: Fluorescence recovery after photobleaching; AUC: Analytical ultracentrifugation; MFA: Marker frequency analysis; crtS: Chr2 replicationtriggering site; t: Half-time recovery of fluorescence

\section{Acknowledgements}

We are grateful to Joaquín Bernal-Bayard, Pedro Escoll-Guerrero, Rocío López-lgual, José Antonio Escudero, and Alexandra Nivina for useful discussions. We thank the technical assistance from Jean Yves Tivenez for assistance and initial observations in FRAP experiments, Laurence Ma and Christiane Bouchier from the Institut Pasteur Genomics Platform for genomic DNA sequencing, and Bertrand Raynal, Sébastien Brulé, and Mounira Tijouani for experimental advice on AUC.

\section{Authors' contributions}

ASB and DM conceived the study. ASB, MEV, OSis, HV, and EK performed the laboratory work. SAP, MGG, OSis, RS and HV were involved in the bioinformatic analysis. ASB, OS, DJC, EPCR, and DM analyzed the data. ASB, SAP, MMG, MEV, OS, EPCR, and DM wrote the paper. All authors read and approved the final manuscript

\section{Funding}

This study was supported by the Institut Pasteur, the Centre National de la Recherche Scientifique (UMR3525), the French National Research Agency 
grants ANR-10-BLAN-131301 (BMC) and ANR-14-CE10-0007 (MAGISBAC), the French Government's Investissement d'Avenir Program, Laboratoire d'Excellence "Integrative Biology of Emerging Infectious Diseases" (ANR-10-LABX-62IBEID to DM), and the Agencia Nacional de Promoción Científica y Tecnológica of Argentina (PICT-2017-0424 to ASB). ASB was supported by an EMBO long-term fellowship (EMBO-ALTF-1473-2010) and Marie Skłodowska-Curie Actions (FP7-PEOPLE-2011-IIF-BMC). ASB, RS, and DJC are Career Members of CONICET. The funders had no role in the study design, data collection and analysis, decision to publish, or preparation of the manuscript.

\section{Availability of data and materials}

All data generated or analyzed during this study are included in this published article, its supplementary information files, and publicly available repositories. The RNA-seq datasets are deposited at the GenBank SRA as BioProject PRJNA509993 [79]. The genomic DNA sequencing data for MFA studies are available at SRA as BioProject PRJNA613768 [80].

\section{Ethics approval and consent to participate}

Not applicable.

\section{Consent for publication}

Not applicable.

\section{Competing interests}

The authors declare that they have no competing interests.

\section{Author details}

${ }^{1}$ Institut Pasteur, Unité Plasticité du Génome Bactérien, UMR3525, CNRS, Paris, France. ${ }^{2}$ Instituto de Investigaciones Biotecnológicas "Dr. Rodolfo A. Ugalde," CONICET - Universidad Nacional de San Martín, San Martín, Buenos Aires, Argentina. ${ }^{3}$ Microbial Evolutionary Genomics, Département Génomes et Génétique, Institut Pasteur, Paris, France. ${ }^{4}$ Centre National de la Recherche Scientifique UMR3525, Paris, France. ${ }^{5}$ Department of Fundamental Microbiology, University of Lausanne, Quartier SORGE, 1003 Lausanne, Switzerland. ${ }^{6}$ Institut Pasteur, Plate-forme Transcriptome et Épigenome, Biomics, Centre d'Innovation et Recherche Technologique (Citech), Paris, France. ${ }^{7}$ Fundación Instituto Leloir, IIBBA-CONICET, Buenos Aires, Argentina. ${ }^{8}$ Department of Science and Environment, Roskilde University, Roskilde, Denmark.

Received: 22 August 2019 Accepted: 31 March 2020

\section{Published online: 29 April 2020}

\section{References}

1. Sclafani RA, Holzen TM. Cell cycle regulation of DNA replication. Annu Rev Genet. 2007:41:237-80

2. Robinson NP, Bell SD. Origins of DNA replication in the three domains of life. FEBS J. 2005;272(15):3757-66.

3. Land M, Hauser L, Jun SR, Nookaew I, Leuze MR, Ahn TH, Karpinets T, Lund O, Kora G, Wassenaar T, et al. Insights from 20 years of bacterial genome sequencing. Functional Integrative Genomics. 2015;15(2):141-61.

4. Touchon $M$, Rocha EP. Coevolution of the organization and structure of prokaryotic genomes. Cold Spring Harb Perspect Biol. 2016;8(1):a018168.

5. Rocha EP. The organization of the bacterial genome. Annu Rev Genet. 2008; 42:211-33.

6. Surovtsev IV, Jacobs-Wagner C. Subcellular organization: a critical feature of bacterial cell replication. Cell. 2018;172(6):1271-93.

7. Rocha EP. Evolutionary patterns in prokaryotic genomes. Curr Opin Microbiol. 2008;11(5):454-60.

8. Lang KS, Merrikh $\mathrm{H}$. The clash of macromolecular titans: replicationtranscription conflicts in bacteria. Annu Rev Microbiol. 2018;72:71-88. https:// doi.org/10.1146/annurev-micro-090817-062514.

9. Esnault E, Valens M, Espeli O, Boccard F. Chromosome structuring limits genome plasticity in Escherichia coli. PLoS Genet. 2007;3(12):e226.

10. Repar J, Warnecke T. Non-random inversion landscapes in prokaryotic genomes are shaped by heterogeneous selection pressures. Mol Biol Evol. 2017:34(8):1902-11.

11. Sobetzko P, Travers A, Muskhelishvili G. Gene order and chromosome dynamics coordinate spatiotemporal gene expression during the bacterial growth cycle. Proc Natl Acad Sci U S A. 2012;109(2):E42-50.
12. Muskhelishvili G, Travers A. Order from the order: how a spatiotemporal genetic program is encoded in a 2-D genetic map of the bacterial chromosome. J Mol Microbiol Biotechnol. 2014;24(5-6):332-43.

13. Gerganova V, Berger M, Zaldastanishvili E, Sobetzko P, Lafon C, Mourez M, Travers A, Muskhelishvili G. Chromosomal position shift of a regulatory gene alters the bacterial phenotype. Nucleic Acids Res. 2015;43(17):8215-26.

14. Slager J, Veening JW. Hard-wired control of bacterial processes by chromosomal gene location. Trends Microbiol. 2016;24(10):788-800.

15. Meyer S, Reverchon S, Nasser W, Muskhelishvili G. Chromosomal organization of transcription: in a nutshell. Curr Genet. 2018;64(3):555-65.

16. Narula J, Kuchina A, Lee DY, Fujita M, Suel GM, Igoshin OA. Chromosomal arrangement of phosphorelay genes couples sporulation and DNA replication. Cell. 2015;162(2):328-37.

17. Brambilla E, Sclavi B. Gene regulation by H-NS as a function of growth conditions depends on chromosomal position in Escherichia coli. G3. 2015; 5(4):605-14

18. Fitzgerald S, Dillon SC, Chao TC, Wiencko HL, Hokamp K, Cameron AD, Dorman CJ. Re-engineering cellular physiology by rewiring high-level global regulatory genes. Sci Rep. 2015;5:17653.

19. Couturier E, Rocha EP. Replication-associated gene dosage effects shape the genomes of fast-growing bacteria but only for transcription and translation genes. Mol Microbiol. 2006;59(5):1506-18.

20. Vieira-Silva S, Rocha EP. The systemic imprint of growth and its uses in ecological (meta)genomics. PLoS Genet. 2010;6(1):e1000808.

21. Bremer H, Dennis PP. Modulation of chemical composition and other parameters of the cell at different exponential growth rates. EcoSal Plus. 2008:3(1). https://doi.org/10.1128/ecosal.5.2.3.

22. Soler-Bistue A, Mondotte JA, Bland MJ, Val ME, Saleh MC, Mazel D. Genomic location of the major ribosomal protein gene locus determines Vibrio cholerae global growth and infectivity. PLoS Genet. 2015;11(4):e1005156.

23. Soler-Bistue A, Timmermans M, Mazel D: The proximity of ribosomal protein genes to oric enhances Vibrio cholerae fitness in the absence of multifork replication. mBio. 2017;8(1). https://doi.org/10.1128/mBio.00097-17.

24. Fournes F, Val M-E, Skovgaard O, Mazel D: Replicate once per cell cycle: replication control of secondary chromosomes. Front Microbiol. 2018; 9(1833). https://doi.org/10.3389/fmicb.2018.01833.

25. Val ME, Marbouty M, de Lemos MF, Kennedy SP, Kemble H, Bland MJ, Possoz C, Koszul R, Skovgaard O, Mazel D. A checkpoint control orchestrates the replication of the two chromosomes of Vibrio cholerae. Sci Adv. 2016; 2(4):e1501914

26. van den Berg J, Boersma AJ, Poolman B. Microorganisms maintain crowding homeostasis. Nat Rev Microbiol. 2017:15(5):309-18.

27. Minton AP. How can biochemical reactions within cells differ from those in test tubes? J Cell Sci. 2015;128(6):1254.

28. Gnutt D, Ebbinghaus $\mathrm{S}$. The macromolecular crowding effect--from in vitro into the cell. Biol Chem. 2016;397(1):37-44.

29. Mourao MA, Hakim JB, Schnell S. Connecting the dots: the effects of macromolecular crowding on cell physiology. Biophys J. 2014;107(12): 2761-6.

30. Scott M, Gunderson CW, Mateescu EM, Zhang Z, Hwa T. Interdependence of cell growth and gene expression: origins and consequences. Science. 2010;330(6007):1099-102.

31. Lipinszki Z, Vernyik V, Farago N, Sari T, Puskas LG, Blattner F, Posfai G, Gyorfy $Z$ : Enhancing the translational capacity of $E$. coli by resolving the codon bias. ACS synthetic biology 2018:7(11):2656-64. https://doi.org/10.1021/ acssynbio.8b00332.

32. Naylor LH. Reporter gene technology: the future looks bright. Biochem Pharmacol. 1999:58(5):749-57.

33. Levin BR, McCall IC, Perrot V, Weiss H, Ovesepian A, Baquero F: A numbers game: ribosome densities, bacterial growth, and antibiotic-mediated stasis and death. mBio. 2017;8(1). https://doi.org/10.1128/mBio.02253-16.

34. Schmid MB, Roth JR. Gene location affects expression level in Salmonella typhimurium. J Bacteriol. 1987;169(6):2872-5.

35. Denks K, Vogt A, Sachelaru I, Petriman NA, Kudva R, Koch HG. The Sec translocon mediated protein transport in prokaryotes and eukaryotes. Mol Membr Biol. 2014:31(2-3):58-84.

36. Skovgaard O, Bak M, Lobner-Olesen A, Tommerup N. Genome-wide detection of chromosomal rearrangements, indels, and mutations in circular chromosomes by short read sequencing. Genome Res. 2011;21(8):1388-93.

37. Forsyth VS, Armbruster CE, Smith SN, Pirani A, Springman AC, Walters MS, Nielubowicz GR, Himpsl SD, Snitkin ES, Mobley HLT: Rapid growth of 
uropathogenic Escherichia coli during human urinary tract infection. mBio. 2018;9(2).

38. Maduike NZ, Tehranchi AK, Wang JD, Kreuzer KN. Replication of the Escherichia coli chromosome in RNase HI-deficient cells: multiple initiation regions and fork dynamics. Mol Microbiol. 2014;91(1):39-56.

39. Powell S, Forslund K, Szklarczyk D, Trachana K, Roth A, Huerta-Cepas J, Gabaldon T, Rattei T, Creevey C, Kuhn M, et al. eggNOG v4.0: nested orthology inference across 3686 organisms. Nucleic Acids Res. 2014; 42(Database issue):D231-9.

40. Kim YE, Hipp MS, Bracher A, Hayer-Hartl M, Hartl FU. Molecular chaperone functions in protein folding and proteostasis. Annu Rev Biochem. 2013;82: 323-55.

41. Kaczanowska M, Ryden-Aulin M. Ribosome biogenesis and the translation process in Escherichia coli. Microbiol Mol Biology Reviews. 2007;71(3):477-94.

42. Delarue M, Brittingham GP, Pfeffer S, Surovtsev IV, Pinglay S, Kennedy KJ, Schaffer M, Gutierrez Jl, Sang D, Poterewicz G, et al. mTORC1 controls phase separation and the biophysical properties of the cytoplasm by tuning crowding. Cell. 2018;174(2):338-49 e320.

43. Akabayov B, Akabayov SR, Lee SJ, Wagner G, Richardson CC. Impact of macromolecular crowding on DNA replication. Nat Commun. 2013;4:1615.

44. Zhou HX. Influence of crowded cellular environments on protein folding, binding, and oligomerization: biological consequences and potentials of atomistic modeling. FEBS Lett. 2013;587(8):1053-61.

45. Fuller RS, Kaguni JM, Kornberg A. Enzymatic replication of the origin of the Escherichia coli chromosome. Proc Natl Acad Sci U S A. 1981;78(12):7370-4.

46. Mika JT, Krasnikov V, van den Bogaart G, de Haan F, Poolman B. Evaluation of pulsed-FRAP and conventional-FRAP for determination of protein mobility in prokaryotic cells. PLoS One. 2011;6(9):e25664.

47. Montero Llopis P, Sliusarenko O, Heinritz J, Jacobs-Wagner C. In vivo biochemistry in bacterial cells using FRAP: insight into the translation cycle. Biophys J. 2012;103(9):1848-59.

48. Wood JM. Bacterial osmoregulation: a paradigm for the study of cellular homeostasis. Annu Rev Microbiol. 2011;65:215-38.

49. Konopka MC, Weisshaar JC, Record MT Jr. Methods of changing biopolymer volume fraction and cytoplasmic solute concentrations for in vivo biophysical studies. Methods Enzymol. 2007;428:487-504.

50. Mika JT, van den Bogaart G, Veenhoff $L$, Krasnikov V, Poolman B. Molecular sieving properties of the cytoplasm of Escherichia coli and consequences of osmotic stress. Mol Microbiol. 2010;77(1):200-7.

51. Slager J, Kjos M, Attaiech L, Veening JW. Antibiotic-induced replication stress triggers bacterial competence by increasing gene dosage near the origin. Cell. 2014;157(2):395-406.

52. Rene $\mathrm{O}$, Alix JH. Late steps of ribosome assembly in E. coli are sensitive to a severe heat stress but are assisted by the HSP70 chaperone machine. Nucleic Acids Res. 2011;39(5):1855-67.

53. Sarkar M, Smith AE, Pielak GJ. Impact of reconstituted cytosol on protein stability. Proc Natl Acad Sci U S A. 2013;110(48):19342-7.

54. Parry BR, Surovtsev IV, Cabeen MT, O'Hern CS, Dufresne ER, Jacobs-Wagner C. The bacterial cytoplasm has glass-like properties and is fluidized by metabolic activity. Cell. 2014;156(1-2):183-94.

55. Miermont A, Waharte F, Hu S, McClean MN, Bottani S, Leon S, Hersen P. Severe osmotic compression triggers a slowdown of intracellular signaling, which can be explained by molecular crowding. Proc Natl Acad Sci U S A. 2013;110(14):5725-30.

56. Val ME, Kennedy SP, Soler-Bistue AJ, Barbe V, Bouchier C, Ducos-Galand M, Skovgaard O, Mazel D. Fuse or die: how to survive the loss of Dam in Vibrio cholerae. Mol Microbiol. 2014;91(4):665-78.

57. Klumpp S, Scott M, Pedersen S, Hwa T. Molecular crowding limits translation and cell growth. Proc Natl Acad Sci U S A. 2013;110(42):16754-9.

58. Record MT Jr, Courtenay ES, Cayley DS, Guttman HJ. Responses of E. coli to osmotic stress: large changes in amounts of cytoplasmic solutes and water. Trends Biochem Sci. 1998;23(4):143-8.

59. Murray $\mathrm{H}$, Koh A. Multiple regulatory systems coordinate DNA replication with cell growth in Bacillus subtilis. PLoS Genet. 2014;10(10):e1004731.

60. Dai X, Zhu M, Warren M, Balakrishnan R, Patsalo V, Okano H, Williamson JR, Fredrick K, Wang YP, Hwa T. Reduction of translating ribosomes enables Escherichia coli to maintain elongation rates during slow growth. Nat Microbiol. 2016;2:16231.

61. Dai X, Zhu M, Warren M, Balakrishnan R, Okano H, Williamson JR, Fredrick K, Hwa T. Slowdown of translational elongation in Escherichia coli under hyperosmotic stress. mBio 2018;9(1):e02375-17. https://doi.org/10.1128/ mBio.02375-17.

62. Lemke JJ, Sanchez-Vazquez P, Burgos HL, Hedberg G, Ross W, Gourse RL. Direct regulation of Escherichia coli ribosomal protein promoters by the transcription factors ppGpp and DksA. Proc Natl Acad Sci U S A. 2011; 108(14):5712-7.

63. Flardh K, Cohen PS, Kjelleberg S. Ribosomes exist in large excess over the apparent demand for protein synthesis during carbon starvation in marine Vibrio sp. strain CCUG 15956. J Bacteriol. 1992;174(21):6780-8.

64. Blazewicz SJ, Barnard RL, Daly RA, Firestone MK. Evaluating rRNA as an indicator of microbial activity in environmental communities: limitations and uses. The ISME journal. 2013;7(11):2061-8.

65. Scott M, Klumpp S, Mateescu EM, Hwa T. Emergence of robust growth laws from optimal regulation of ribosome synthesis. Mol Syst Biol. 2014;10:747.

66. Jun S, Si F, Pugatch R, Scott M. Fundamental principles in bacterial physiology-history, recent progress, and the future with focus on cell size control: a review. Reports Progress Physics Physical Soc. 2018;81(5):056601.

67. Hall BG, Acar H, Nandipati A, Barlow M. Growth rates made easy. Mol Biol Evol. 2014;31(1):232-8.

68. Rivera MC, Maguire B, Lake JA. Isolation of ribosomes and polysomes. Cold Spring Harb Protoc. 2015;2015(3):293-9.

69. Vercruysse M, Kohrer C, Davies BW, Arnold MF, Mekalanos JJ, RajBhandary UL, Walker GC. The highly conserved bacterial RNase YbeY is essential in Vibrio cholerae, playing a critical role in virulence, stress regulation, and RNA processing. PLoS Pathog. 2014;10(6):e1004175.

70. Krin E, Pierle SA, Sismeiro O, Jagla B, Dillies MA, Varet H, Irazoki O, Campoy $S$, Rouy Z, Cruveiller $S$, et al. Expansion of the SOS regulon of Vibrio cholerae through extensive transcriptome analysis and experimental validation. BMC Genomics. 2018;19(1):373.

71. Langmead B, Trapnell C, Pop M, Salzberg SL. Ultrafast and memory-efficient alignment of short DNA sequences to the human genome. Genome Biol. 2009;10(3):R25.

72. Anders S, Pyl PT, Huber W. HTSeq--a Python framework to work with highthroughput sequencing data. Bioinformatics. 2015;31(2):166-9.

73. Love Ml, Huber W, Anders S. Moderated estimation of fold change and dispersion for RNA-seq data with DESeq2. Genome Biol. 2014;15(12):550.

74. Benjamini $Y$, Hochberg Y. Controlling the false discovery rate - a practical and powerful approach to multiple testing. J Roy Stat Soc B Met. 1995;57(1): 289-300.

75. Varet H, Brillet-Gueguen L, Coppee JY, Dillies MA. SARTools: a DESeq2- and EdgeR-based R pipeline for comprehensive differential analysis of RNA-seq data. PLoS One. 2016;11(6):e0157022.

76. Krzywinski M, Schein J, Birol I, Connors J, Gascoyne R, Horsman D, Jones SJ, Marra MA. Circos: an information aesthetic for comparative genomics. Genome Res. 2009;19(9):1639-45.

77. Team RC. The R project for statistical computing. In: The R project for statistical computing. Viena: R Foundation for Statistical Computing; 2014.

78. Pierle SA, Dark MJ, Dahmen D, Palmer GH, Brayton KA. Comparative genomics and transcriptomics of trait-gene association. BMC Genomics. 2012;13:669.

79. Soler-Bistué AA-PS, Garcia-Garcerá M, Val ME, Sismeiro O, Varet H, Sieira R, Krin E, Skovgaard O, Comerci DJ, Rocha EPC, Didier Mazel D.

Macromolecular crowding links ribosomal protein gene dosage to growth rate in Vibrio cholerae - BioProject. In The Sequence Read Archive. https:// www.ncbi.nlm.nih.gov/sra/PRJNA509993.

80. Soler-Bistué A A-PS, Garcia-Garcerá M, Val ME, Sismeiro O, Varet H,Sieira R, Krin E, Skovgaard O, Comerci DJ, Rocha EPC, and Didier Mazel D: Marker frequency analysis of Vibrio cholerae s10-spc-alpha movants in fast growing conditions. . In: The Sequence Read Archive (https://www.ncbi.nlm.nih.gov/ sra/PRJNA613768)

81. Mazel D. Integrons: agents of bacterial evolution. Nat Rev Microbiol. 2006; 4(8):608-20.

\section{Publisher's Note}

Springer Nature remains neutral with regard to jurisdictional claims in published maps and institutional affiliations. 